\title{
The Ubiquitous Imprint of Radiative Acceleration in the Mean Absorption Spectrum of Quasar Outflows
}

\author{
Lluís Mas-Ribas $^{1,2,3}$ (D) and Renate Mauland ${ }^{3}$ (D) \\ ${ }^{1}$ Jet Propulsion Laboratory, California Institute of Technology, 4800 Oak Grove Drive, Pasadena, CA 91109, USA; 1luis.mas-ribas@jpl.nasa.gov \\ ${ }^{2}$ California Institute of Technology, 1200 E. California Boulevard, Pasadena, CA 91125, USA \\ ${ }^{3}$ Institute of Theoretical Astrophysics, University of Oslo, Postboks 1029, NO-0315 Oslo, Norway \\ Received 2018 December 28; revised 2019 September 17; accepted 2019 October 16; published 2019 December 3
}

\begin{abstract}
Observational evidence revealing the main mechanisms that accelerate quasar outflows has proven difficult to obtain due to the complexity of the absorption features that this gas produces in the spectra of the emission sources. We build 36 composite outflow spectra, covering a large range of outflow and quasar parameters, by stacking broad $\left(>450 \mathrm{~km} \mathrm{~s}^{-1}\right)$ absorption line systems in the spectra of SDSS-III/BOSS DR12 quasars. The two lines of the atomic doublet of C IV, with a separation of $\approx 497 \mathrm{~km} \mathrm{~s}^{-1}$, as well as those of other species, appear well resolved in most of our composites. This agrees with broad outflow troughs consisting of the superposition of narrow absorbers. We also report on the ubiquitous detection of the radiative-acceleration signature known as line-locking in all our composite outflow spectra, including one spectrum that was strictly built from broad absorption line (BAL) systems. This is the first line-locking detection in BAL composite spectra. Line-locking is driven by the $\mathrm{C}$ IV atomic doublet and is visible on the blue side of most strong absorption transitions. Similar effects from the doublets of O VI, Si IV, or N V, however, seem to not be present. Our results confirm that radiation pressure is a prevalent mechanism for accelerating outflows in quasars.
\end{abstract}

Unified Astronomy Thesaurus concepts: Active galactic nuclei (1317); AGN host galaxies (2017); Galaxy formation (595); Interstellar scattering (854); Quasar absorption line spectroscopy (1317); Broad-absorption line quasar (183)

\section{Introduction}

Supermassive black holes, with masses as high as billions of times the mass of the Sun, inhabit the center of most if not all massive galaxies in the universe. Although their size is smaller than that of their hosts, these formidable objects can determine the fate of the whole galaxy (Furlanetto \& Loeb 2001; Scannapieco \& Oh 2004; Haiman \& Bryan 2006), as well as contribute to the metal enrichment of the distant intergalactic medium (Cavaliere et al. 2002; Levine \& Gnedin 2005).

When supermassive black holes grow by undergoing episodes of gas accretion, they release large amounts of energy and become detectable as active galactic nuclei (AGNs). One of their brightest phases is represented by the so-called quasars, and the energy release, referred to as AGN or quasar feedback, results in powerful winds that can expel material out of the host galaxy (see the reviews by King \& Pounds 2015; Harrison 2017).

The origin of quasar feedback may reside in the outflows launched from the innermost regions around the black holes, which can reach velocities of up to $10 \%-20 \%$ of the speed of light (Moe et al. 2009; Harrison et al. 2018). However, probing the link between fast outflows and quasar feedback is challenging because the mechanisms driving these outflows are still unclear and simulations cannot cover the whole range of physical scales and processes involved (Ciotti et al. 2010; Hopkins \& Elvis 2010; Barnes et al. 2018). It is broadly believed that outflows can be accelerated by the pressure that radiation from the quasar exerts on the gas, but questions concerning the overionization, confinement, and stability of the outflowing gas have been puzzling astronomers for decades (Williams 1972; de Kool \& Begelman 1995; Murray et al. 1995; Proga et al. 2000; Proga 2007b; Baskin et al. 2014;
Proga \& Waters 2015; Matthews et al. 2016; Waters \& Proga 2016; Bianchi et al. 2019).

The theory of radiative acceleration in quasar outflows was initially developed by Mushotzky et al. (1972), Scargle (1973), Burbidge \& Burbidge (1975), Braun \& Milgrom (1989), and Arav \& $\mathrm{Li}$ (1994), and was inspired by the theory that was proposed for the ejection of material from massive stars by Milne (1926) and Lucy \& Solomon (1970). A common feature appearing in these works is the phenomenon known as linelocking, for which absorption systems in the outflow become bound to a fixed relative velocity that coincides with the velocity separation of two atomic transitions, typically the two lines of an atomic doublet. In detail, the outflow absorbs (or scatters) radiation from the quasar and thus becomes accelerated. This occurs for both line and continuum quasar radiation, although the absorption of line radiation injects a larger boost than the continuum because the flux in the emission lines is highest. Regarding now the case of an atomic doublet, a region absorbing radiation from both lines of the doublet can suffer a reduction in the acceleration if another region between the first region and the source moves at a lower (relative) speed coinciding with the doublet separation. In this scenario, the region in the middle also absorbs radiation from the two lines of the doublet in its frame, and given the relative velocity between the two regions, this means that it absorbs the flux that was previously available for the red line of the front region. As the back region absorbs more flux and increases its speed, the flux-masking disappears and the front region can again absorb the two lines and increase its speed accordingly, thus restoring the previous relative velocity. The acceleration of the front region is thus regulated by the back region, and the two regions are eventually locked in a metastable state where the relative velocity corresponds to the doublet separation. 
The observation of line-locking signatures of a given species in the spectra of quasars can therefore confirm the presence of radiative acceleration in the outflows. These observations, however, are not easy due to the complexity of the outflow absorption features, which are usually broad, and also because it is difficult to distinguish line-locking from randomly located absorption components (e.g., Korista et al. 1993).

Absorption features due to outflows were first identified as strong and broad absorption lines (BALs) from high-ionization species such as C IV, Si IV, or N V, blueshifted from the AGN rest-frame position (Lynds 1967). Today, outflow features are observed in a variety of widths and depths, and line-locking from high-ionization species is suggested in a small number of them: Foltz et al. (1987) and Srianand et al. (2002) reported linelocking features in the spectra of one quasar each, and other tentative detections in a few quasars were presented in, e.g., Srianand (2000), Ganguly et al. (2003), Gupta et al. (2003), North et al. (2006), and Lu \& Lin (2018b). Furthermore, linelocking was also detected by Bowler et al. (2014), who analyzed composite spectra of narrow $\left(\leqslant 200 \mathrm{~km} \mathrm{~s}^{-1}\right)$ C IV absorption lines in the spectra of quasars. These authors analyzed narrow absorbers in quasars containing BALs, but they did not use the BALs themselves in order to be able to resolve the C IV doublets and detect the line-locking signatures.

Although the total width of the outflow troughs can be as large as $\sim 30,000 \mathrm{~km} \mathrm{~s}^{-1}$, they sometimes consist of the superposition of narrower features, of approximately a few hundred $\mathrm{km} \mathrm{s}^{-1}$, the so-called narrow absorption lines (NALs; e.g., Gabel et al. 2006; Lu \& Lin 2018a, 2018b). Therefore, it should be possible to obtain the narrow outflow absorption spectrum when the broad troughs are centered at a characteristic position when the composite spectra are created (Perrotta et al. 2018). Given this consideration, we conduct here a search for the line-locking signature in 36 composite spectra of broad $\left(>450 \mathrm{~km} \mathrm{~s}^{-1}\right)$ C IV absorption line systems (including BALs) representing outflows, and test the prevalence of line-locking for multiple properties of the outflows, the quasars, and different atomic species. We assess and present the physical properties of the outflow inferred from the analysis of the composite spectra in our companion paper, Mas-Ribas (2019).

In Sections 2 and 3 we detail the data and methods, respectively, and in Section 4 we analyze one of our composite outflow spectra in detail. The C IV, O VI, and N V line-locking features are assessed in Section 5. We discuss our findings in Section 6 before we conclude in Section 7.

We assume a flat $\Lambda$ CDM cosmology with the parameter values from Planck Collaboration et al. (2016).

\section{Data}

We detail in Section 2.1 the quasar spectra we used in our calculations, and present the atomic data for the analysis of the absorption lines in Section 2.2.

\subsection{Quasar Spectra}

We use the 297301 quasar spectra in the twelfth data release of the SDSS-III/BOSS (Eisenstein et al. 2011; Dawson et al. 2013) quasar catalog, DR12Q (Pâris et al. 2017). The characteristics of the Sloan Digital Sky Survey (SDSS) telescope and camera are detailed in Gunn et al. (1998, 2006)

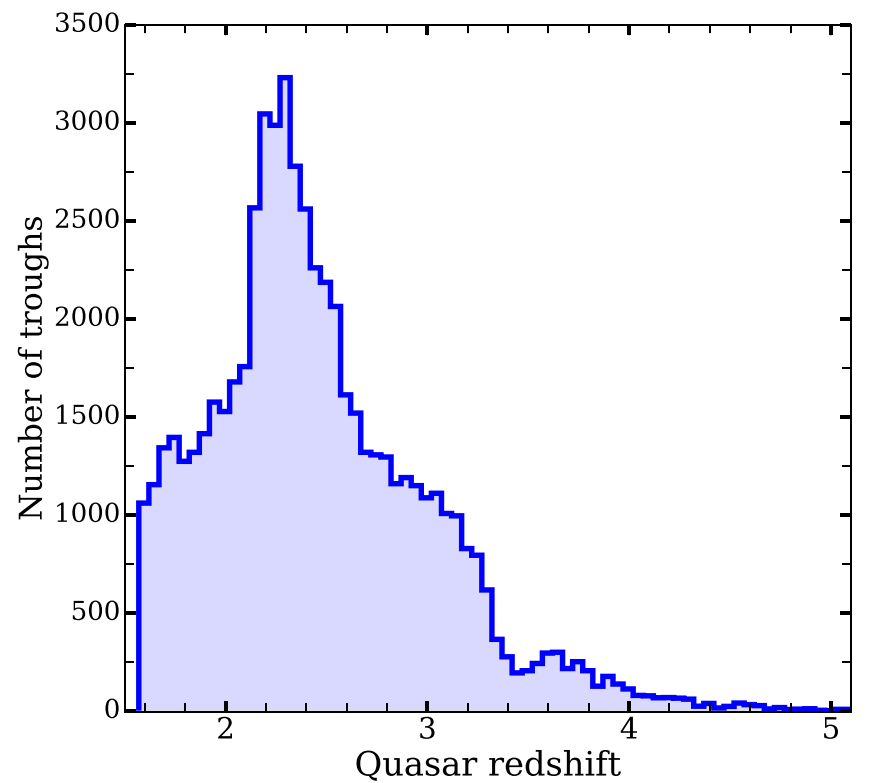

Figure 1. Redshift distribution of the DR12Q quasars containing broad absorption lines, covering the range $1.5 \lesssim z \lesssim 5.5$.

and Ross et al. (2012), and the SDSS/BOSS spectrographs in Smee et al. (2013).

We also use the DR12QBAL catalog, which compiles the individual properties of the $\mathrm{C}$ IV absorption troughs with widths $>450 \mathrm{~km} \mathrm{~s}^{-1}$ detected in the spectra of the DR12Q quasars. The DR12QBAL catalog contains 63,549 troughs with measurements of their position, width, and maximum depth. The position of each trough was set at the velocity distance from the quasar where the flux within the absorption trough is the minimum, and the trough width was defined as the velocity range in which the measured normalized quasar flux density (i.e., the flux transmission) is lower than 0.9 (Pâris et al. 2012, 2017). We remove from further analysis 3677 objects $(\simeq 83 \%$ of them with velocity offset from the quasar below $\left.v \sim 400 \mathrm{~km} \mathrm{~s}^{-1}\right)$. The position of the minimum flux within these troughs is quoted in the catalog outside the boundaries of the troughs. The composite spectrum of these discarded troughs does not show significant absorption features, indicating that they are false positives and/ or severely affected by incorrect velocity offset measurements or noise, and their inclusion in the samples would simply increase the noise at low outflow velocities. Our final sample thus consists of 59,872 absorption troughs.

Figure 1 shows the redshift distribution of the DR12Q quasars containing troughs, which spans the range $1.5 \leqslant z \leqslant$ 5.5 , although only a small number of objects have values above $z \sim 3.3$. The distribution peaks at $z \sim 2.4$, similar to its mean value of $\bar{z}=2.46$, and decreases rapidly toward both lower and higher values. The upper panel in Figure 2 shows the distribution of the velocity offset of the troughs from the quasars, i.e., the outflow velocities, for the final trough sample. The distribution peaks at around $2000 \mathrm{~km} \mathrm{~s}^{-1}$ and decreases toward higher velocities up to $25,000 \mathrm{~km} \mathrm{~s}^{-1}$, with a slight change of slope that flattens beyond $\sim 12,000 \mathrm{~km} \mathrm{~s}^{-1}$. For outflow velocities lower than that of the peak, the decrease in the number of troughs is much steeper, flattening at velocities below $\sim 1000 \mathrm{~km} \mathrm{~s}^{-1}$. Overall, this distribution is similar to that obtained by Nestor et al. (2008), who searched for C IV troughs in the quasar spectra of the fourth SDSS data release. The lower panel in Figure 2 displays the distribution of trough widths, 

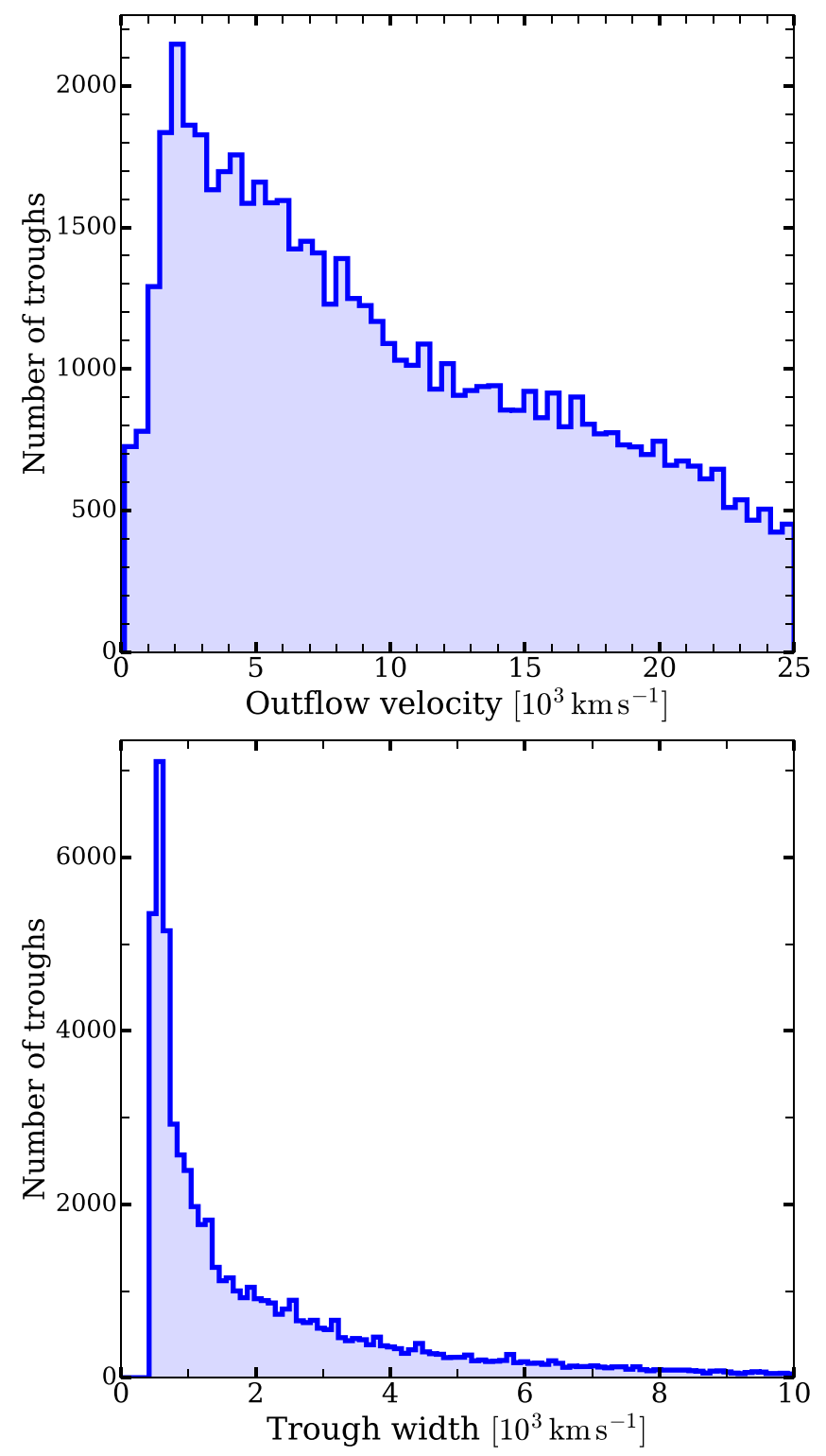

Figure 2. Top panel: Distribution of outflow velocities, with a minimum value of $\sim 200 \mathrm{~km} \mathrm{~s}^{-1}$ and a peak at $\sim 2000 \mathrm{~km} \mathrm{~s}^{-1}$, decreasing toward higher velocities. Bottom panel: Distribution of trough widths, peaking at around $700 \mathrm{~km} \mathrm{~s}^{-1}$, and decreasing rapidly toward larger widths. The distribution spans from $\sim 300$ to $25,000 \mathrm{~km} \mathrm{~s}^{-1}$, but we only plot values below $10,000 \mathrm{~km} \mathrm{~s}^{-1}$ due to the small number of objects above this threshold.

which peaks at $\sim 700 \mathrm{~km} \mathrm{~s}^{-1}$ and decreases rapidly toward higher and lower values. The plot covers the interval $\simeq 300-10,000 \mathrm{~km} \mathrm{~s}^{-1}$, although a small number of troughs have widths of up to $\sim 25,000 \mathrm{~km} \mathrm{~s}^{-1}$.

\subsection{Absorption Lines}

Table 1 presents vacuum atomic data in the rest frame between 500 and $3000 \AA$ relevant for the analysis of outflow absorption lines. We have built this catalog by compiling data from the database LINETOOLS $^{4}$ (Prochaska et al. 2016) and that of the National Institute of Standards and Technology (NIST ${ }^{5}$; Kramida et al.). This list includes a number of strong absorption lines from species that are typically not observed

\footnotetext{
4 https://linetools.readthedocs.io/en/latest/

5 https://www.nist.gov/pml/atomic-spectra-database
}

Table 1

Atomic Data

\begin{tabular}{|c|c|c|c|}
\hline Transition & $\lambda(\AA)$ & $f$ & $\overline{\text { Reference }}$ \\
\hline O III $\lambda 507$ & 507.3910 & 0.18500 & 1 \\
\hline O III $\lambda 533$ & 533.5099 & 0.29899 & 1 \\
\hline O II $\lambda 539$ & 539.0855 & 0.06520 & 1 \\
\hline $\mathrm{Ne}$ IV $\lambda 541$ & 541.1270 & 0.03900 & 1 \\
\hline Ne IV $\lambda 542$ & 542.0730 & 0.07789 & 1 \\
\hline C II $\lambda 543$ & 543.2570 & 0.03489 & 1 \\
\hline $\mathrm{Ne}$ IV $\lambda 543$ & 543.8910 & 0.11599 & 1 \\
\hline O IV $\lambda 553$ & 553.3300 & 0.11200 & 1 \\
\hline O IV $\lambda 554$ & 554.0750 & 0.22400 & 1 \\
\hline $\mathrm{Ne}$ VI $\lambda 558$ & 558.5900 & 0.09070 & 1 \\
\hline $\mathrm{Ne}$ VI $\lambda 558$ & 558.5900 & 0.09070 & 1 \\
\hline C II $\lambda 560$ & 560.2394 & 0.05710 & 1 \\
\hline $\mathrm{Ne} V \lambda 568$ & 568.4200 & 0.09279 & 1 \\
\hline He I $\lambda 584$ & 584.3300 & 0.27625 & 2 \\
\hline C II $\lambda 594$ & 594.8000 & 0.11699 & 1 \\
\hline O IV $\lambda 608$ & 608.3980 & 0.06700 & 1 \\
\hline $\operatorname{Mg} \times \lambda 609$ & 609.7900 & 0.08420 & 1 \\
\hline $\operatorname{Mg} \times \lambda 624$ & 624.9500 & 0.04100 & 1 \\
\hline O IV $\lambda 625$ & 625.0000 & 0.12500 & 2 \\
\hline $\mathrm{O} v \lambda 629$ & 629.7300 & 0.51499 & 1 \\
\hline S II $\lambda 641$ & 641.7670 & 0.25999 & 1 \\
\hline S IV $\lambda 657$ & 657.3280 & 1.13000 & 2 \\
\hline S IV $\lambda 661$ & 661.4430 & 1.02000 & 2 \\
\hline S III $\lambda 680$ & 680.6800 & 1.38000 & 2 \\
\hline S III $\lambda 681$ & 681.4700 & 0.06830 & 1 \\
\hline S III $\lambda 683$ & 683.5860 & 1.34000 & 2 \\
\hline N III $\lambda 684$ & 684.9960 & 0.13500 & 1 \\
\hline N III $\lambda 685$ & 685.8200 & 0.32000 & 2 \\
\hline S III $\lambda 698$ & 698.7310 & 0.78299 & 1 \\
\hline O III $\lambda 702$ & 702.3320 & 0.13699 & 1 \\
\hline S III $\lambda 724$ & 724.2890 & 0.35199 & 1 \\
\hline S IV $\lambda 744$ & 744.9070 & 0.25099 & 1 \\
\hline S IV $\lambda 748$ & 748.4000 & 0.50000 & 1 \\
\hline N III $\lambda 763$ & 763.3400 & 0.08200 & 1 \\
\hline S II $\lambda 763$ & 763.6570 & 0.39800 & 1 \\
\hline S II $\lambda 764$ & 764.4200 & 0.79500 & 1 \\
\hline N IV $\lambda 765$ & 765.1480 & 0.61599 & 1 \\
\hline S II $\lambda 765$ & 765.6930 & 1.19000 & 1 \\
\hline Ne VIII $\lambda 770$ & 770.4090 & 0.10300 & 1 \\
\hline Ne VIII $\lambda 780$ & 780.3240 & 0.05050 & 1 \\
\hline S V $\lambda 786$ & 786.4700 & 1.36000 & 2 \\
\hline O IV $\lambda 787$ & 787.7110 & 0.11100 & 1 \\
\hline O I $\lambda 791$ & 791.9732 & 0.04639 & 1 \\
\hline S IV $\lambda 809$ & 809.6680 & 0.10400 & 1 \\
\hline S IV $\lambda 815$ & 815.9450 & 0.08500 & 2 \\
\hline O II $\lambda 832$ & 832.7572 & 0.04439 & 1 \\
\hline O III $\lambda 832$ & 832.9270 & 0.10700 & 1 \\
\hline O II $\lambda 833$ & 833.3294 & 0.08860 & 1 \\
\hline O II $\lambda 834$ & 834.4655 & 0.13199 & 1 \\
\hline Fe III $\lambda 844$ & 844.2880 & 0.06840 & 1 \\
\hline C II $\lambda 858$ & 858.0918 & 0.01300 & 1 \\
\hline Fe III $\lambda 859$ & 859.7230 & 0.11500 & 1 \\
\hline O I $\lambda 877 a$ & 877.7983 & 0.01970 & 1 \\
\hline O I $\lambda 877 b$ & 877.8787 & 0.05889 & 1 \\
\hline Si II $\lambda 889$ & 889.7228 & 0.04340 & 1 \\
\hline S II $\lambda 906$ & 906.8850 & 0.21000 & 1 \\
\hline P III $\lambda 913$ & 913.9683 & 0.20300 & 1 \\
\hline O III $\lambda 915$ & 915.6131 & 0.15900 & 1 \\
\hline P III $\lambda 917$ & 917.1178 & 0.40400 & 1 \\
\hline $\mathrm{S}$ VI $\lambda 933$ & 933.3780 & 0.43700 & 1 \\
\hline Н I $\lambda 937$ & 937.8034 & 0.00780 & 1 \\
\hline $\mathrm{S}$ VI $\lambda 944$ & 944.5230 & 0.21500 & 1 \\
\hline C I $\lambda 945$ & 945.1910 & 0.15200 & 1 \\
\hline O I $\lambda 948$ & 948.6855 & 0.00631 & 1 \\
\hline H I $\lambda 949$ & 949.7430 & 0.01395 & 1 \\
\hline
\end{tabular}


Table 1

(Continued)

\begin{tabular}{|c|c|c|c|}
\hline Transition & $\lambda(\AA)$ & $f$ & Reference \\
\hline $\mathrm{P}$ IV $\lambda 950$ & 950.6600 & 1.60000 & 2 \\
\hline N I $\lambda 963$ & 963.9903 & 0.01240 & 1 \\
\hline N I $\lambda 964$ & 964.6256 & 0.00790 & 1 \\
\hline H I $\lambda 972$ & 972.5367 & 0.02901 & 1 \\
\hline C III $\lambda 977$ & 977.0201 & 0.75700 & 1 \\
\hline O I $\lambda 988$ & 988.7734 & 0.04650 & 1 \\
\hline N III $\lambda 989$ & 989.7990 & 0.12300 & 1 \\
\hline Si II $\lambda 989$ & 989.8731 & 0.17100 & 1 \\
\hline S III $\lambda 1012$ & 1012.495 & 0.04380 & 1 \\
\hline Si II $\lambda 1020$ & 1020.698 & 0.01680 & 1 \\
\hline H I $\lambda 1025$ & 1025.722 & 0.07914 & 1 \\
\hline O I $\lambda 1027$ & 1027.431 & 0.01962 & 2 \\
\hline O I $\lambda 1028$ & 1028.157 & 0.02010 & 2 \\
\hline O VI $\lambda 1031$ & 1031.926 & 0.13250 & 1 \\
\hline C II $\lambda 1036$ & 1036.336 & 0.11800 & 1 \\
\hline C II $\lambda 1037$ & 1037.018 & 0.11800 & 2 \\
\hline O VI $\lambda 1037$ & 1037.616 & 0.06580 & 1 \\
\hline O I $\lambda 1039$ & 1039.230 & 0.00907 & 1 \\
\hline Fe II $\lambda 1055$ & 1055.261 & 0.00750 & 1 \\
\hline S IV $\lambda 1062$ & 1062.664 & 0.04940 & 1 \\
\hline $\mathrm{S} \mathrm{IV}^{*} \lambda 1073$ & 1072.962 & 0.04200 & 2 \\
\hline Fe II $\lambda 1081$ & 1081.874 & 0.01260 & 1 \\
\hline O III $\lambda 1083$ & 1083.993 & 0.11100 & 1 \\
\hline Fe II $\lambda 1096$ & 1096.876 & 0.03200 & 1 \\
\hline Fe II $\lambda 1112$ & 1112.048 & 0.00620 & 1 \\
\hline C I $\lambda 1112$ & 1112.269 & 0.01610 & 1 \\
\hline $\mathrm{P} \vee \lambda 1117$ & 1117.977 & 0.47200 & 1 \\
\hline Fe II $\lambda 1121$ & 1121.974 & 0.02020 & 1 \\
\hline C I $\lambda 1122$ & 1122.437 & 0.00511 & 1 \\
\hline Fe III $\lambda 1122$ & 1122.524 & 0.05440 & 1 \\
\hline Fe II $\lambda 1125$ & 1125.447 & 0.01600 & 1 \\
\hline $\mathrm{P} \vee \lambda 1128$ & 1128.007 & 0.23300 & 1 \\
\hline C I $\lambda 1129$ & 1129.195 & 0.00771 & 1 \\
\hline Fe II $\lambda 1133$ & 1133.665 & 0.00550 & 1 \\
\hline N I $\lambda 1134 a$ & 1134.165 & 0.01460 & 1 \\
\hline N I $\lambda 1134 b$ & 1134.414 & 0.02870 & 1 \\
\hline $\mathrm{N}$ I $\lambda 1134 c$ & 1134.980 & 0.04160 & 1 \\
\hline Fe II $\lambda 1143$ & 1143.226 & 0.01920 & 1 \\
\hline Fe II $\lambda 1144$ & 1144.937 & 0.08300 & 1 \\
\hline C I $\lambda 1158$ & 1158.324 & 0.00655 & 1 \\
\hline $\mathrm{C}$ III $^{*} \lambda 1175$ & 1175.260 & 0.27240 & 2 \\
\hline S III $\lambda 1190$ & 1190.203 & 0.02370 & 1 \\
\hline Si II $\lambda 1190$ & 1190.415 & 0.29200 & 1 \\
\hline C I $\lambda 1193$ & 1193.030 & 0.04090 & 1 \\
\hline Si II $\lambda 1193$ & 1193.289 & 0.58200 & 1 \\
\hline Si II $\lambda 1194$ & 1194.500 & 0.73700 & 2 \\
\hline Mn II $\lambda 1197$ & 1197.184 & 0.21700 & 1 \\
\hline Mn II $\lambda 1199$ & 1199.391 & 0.16900 & 1 \\
\hline N I $\lambda 1199$ & 1199.549 & 0.13200 & 1 \\
\hline N I $\lambda 1200$ & 1200.223 & 0.08690 & 1 \\
\hline N I $\lambda 1200 b$ & 1200.709 & 0.04320 & 1 \\
\hline Si III $\lambda 1206$ & 1206.500 & 1.63000 & 1 \\
\hline H I $\lambda 1215$ & 1215.670 & 0.41640 & 1 \\
\hline N V $\lambda 1238$ & 1238.821 & 0.15600 & 1 \\
\hline N V $\lambda 1242$ & 1242.804 & 0.07770 & 1 \\
\hline S II $\lambda 1250$ & 1250.578 & 0.00543 & 1 \\
\hline S II $\lambda 1253$ & 1253.805 & 0.01090 & 1 \\
\hline Si II $\lambda 1260$ & 1260.422 & 1.18000 & 1 \\
\hline Si II $\lambda 1264$ & 1264.730 & 1.09000 & 2 \\
\hline C I $\lambda 1276$ & 1276.482 & 0.00589 & 1 \\
\hline C I $\lambda 1277$ & 1277.245 & 0.08530 & 1 \\
\hline O I $\lambda 1302$ & 1302.168 & 0.04800 & 1 \\
\hline Si II $\lambda 1304$ & 1304.370 & 0.08630 & 1 \\
\hline Ni II $\lambda 1317$ & 1317.217 & 0.14599 & 1 \\
\hline
\end{tabular}

Table 1

(Continued)

\begin{tabular}{|c|c|c|c|}
\hline Transition & $\lambda(\AA)$ & $f$ & Reference \\
\hline$\overline{C \text { I } \lambda 1328}$ & 1328.833 & 0.07580 & 1 \\
\hline C II $\lambda 1334$ & 1334.532 & 0.12800 & 1 \\
\hline C II $\lambda 1335$ & 1335.708 & 0.11500 & 2 \\
\hline Ni II $\lambda 1370$ & 1370.132 & 0.07690 & 1 \\
\hline Si IV $\lambda 1393$ & 1393.755 & 0.52399 & 1 \\
\hline Si IV $\lambda 1402$ & 1402.770 & 0.25999 & 1 \\
\hline Ni II $\lambda 1454$ & 1454.842 & 0.03230 & 1 \\
\hline Si II $\lambda 1526$ & 1526.707 & 0.12700 & 1 \\
\hline C IV $\lambda 1548$ & 1548.204 & 0.18999 & 1 \\
\hline C IV $\lambda 1550$ & 1550.770 & 0.09520 & 1 \\
\hline C I $\lambda 1560$ & 1560.309 & 0.07740 & 1 \\
\hline Si I $\lambda 1589$ & 1589.174 & 0.05040 & 1 \\
\hline Fe II $\lambda 1608$ & 1608.451 & 0.05770 & 1 \\
\hline C I $\lambda 1656$ & 1656.928 & 0.14900 & 1 \\
\hline Al II $\lambda 1670$ & 1670.788 & 1.74000 & 1 \\
\hline Ni II $\lambda 1703$ & 1703.411 & 0.00600 & 1 \\
\hline Ni II $\lambda 1709$ & 1709.604 & 0.03240 & 1 \\
\hline Ni II $\lambda 1741$ & 1741.553 & 0.04270 & 1 \\
\hline Ni II $\lambda 1751$ & 1751.915 & 0.02770 & 1 \\
\hline Ni II $\lambda 1773$ & 1773.949 & 0.00621 & 1 \\
\hline Ni II $\lambda 1804$ & 1804.473 & 0.00716 & 1 \\
\hline $\operatorname{Mg}$ I $\lambda 1827$ & 1827.935 & 0.02420 & 1 \\
\hline Al III $\lambda 1854$ & 1854.716 & 0.57499 & 1 \\
\hline Al III $\lambda 1862$ & 1862.789 & 0.28600 & 1 \\
\hline Zn II $\lambda 2026$ & 2026.137 & 0.50100 & 1 \\
\hline Cr II $\lambda 2056$ & 2056.256 & 0.10300 & 1 \\
\hline Cr II $\lambda 2062$ & 2062.234 & 0.10499 & 1 \\
\hline Zn II $\lambda 2062$ & 2062.664 & 0.25299 & 1 \\
\hline Cr II $\lambda 2066$ & 2066.161 & 0.06979 & 1 \\
\hline Fe II $\lambda 2344$ & 2344.213 & 0.11400 & 1 \\
\hline Fe II $\lambda 2374$ & 2374.461 & 0.03130 & 1 \\
\hline Fe II $\lambda 2382$ & 2382.765 & 0.32000 & 1 \\
\hline Mn II $\lambda 2576$ & 2576.877 & 0.36100 & 1 \\
\hline $\mathrm{Fe}$ II $\lambda 2586$ & 2586.650 & 0.06910 & 1 \\
\hline Mn II $\lambda 2594$ & 2594.499 & 0.28000 & 1 \\
\hline $\mathrm{Fe}$ II $\lambda 2600$ & 2600.172 & 0.23900 & 1 \\
\hline Mn II $\lambda 2606$ & 2606.462 & 0.19800 & 1 \\
\hline Mg II $\lambda 2796$ & 2796.354 & 0.61550 & 1 \\
\hline Mg II $\lambda 2803$ & 2803.531 & 0.30580 & 1 \\
\hline $\operatorname{Mg}$ I $\lambda 2852$ & 2852.964 & 1.73000 & 1 \\
\hline
\end{tabular}

Note. Transitions arising from excited metastable states are denoted by an asterisk.

References: (1) LINETOOLS (2) NIST.

in interstellar or intergalactic gas studies but that may arise under the extreme conditions of high temperature, density, and strong radiation fields present in quasar outflows. Many of these lines originate from excited metastable states above the ground level. However, this set of lines is not complete, and the interested reader is referred to the NIST for the full spectroscopic data. The first column in Table 1 denotes each transition, and the second colum lists its line center in Angstrom (in the vacuum). The corresponding oscillator strengths are shown in the third column, and the reference source is presented in the fourth column.

This table is publicly available in digital format at https:// github.com/lluism/BALs and the access is open for the community to contribute to updating, correcting, and/or extending it to make it useful for future studies. 


\section{Methods}

We detail the calculation of the mean quasar spectrum in Section 3.1 and that of the outflow composite spectrum in Section 3.2. Section 3.3 describes the method for modeling the absorption lines.

\subsection{Mean Quasar Spectrum Calculation}

We calculate a weighted-mean quasar spectrum that is used to normalize the spectra for the computation of the outflow composite spectrum, following our procedure in Mas-Ribas et al. (2017). Below we summarize the main steps of this calculation and refer the interested reader to the aforementioned work for details.

In our calculations, we do not consider spectral pixels that are affected by the skylines reported by Palanque-Delabrouille et al. (2013). Furthermore, we always correct the flux in the Ly $\alpha$ forest of the quasar spectra for the redshift-dependent average absorption in this region, using the analytical formula proposed by Faucher-Giguère et al. (2008b; Equation (7) in Mas-Ribas et al. 2017). For the computation of the mean spectrum we use all the spectra in the DR12Q catalog. This is because the coincident position of troughs in the quasar spectra yields an undesired average broad absorption feature next to the quasar emission lines when only quasars with broad troughs are considered.

The calculation of the mean quasar spectrum is performed as follows: we shift the observed spectra to the rest-frame position of the quasars, considering the visual inspection quasar redshift values in DR12Q, and rebin the flux density and error into an evenly spaced wavelength array with a pixel width of $1 \AA$. The spectra are then normalized by dividing them by their corresponding mean flux in the wavelength windows free of emission lines $1300<\lambda_{\mathrm{r}} / \AA<1383$ and $1408<\lambda_{\mathrm{r}} / \AA<$ 1500. When more than $20 \%$ of the pixels in these intervals for one spectrum are not accounted for due to skylines, this spectrum is not used. This constraint rejects less than $10 \%$ of the spectra in the catalog.

A mean signal-to-noise ratio $(\mathrm{S} / \mathrm{N})$ is then calculated for each remaining spectrum $j$ as

$$
\mathrm{S} / \mathrm{N}_{j}=\frac{\sum_{i} f_{i j} / N_{j}}{\left(\sum_{i} e_{i j}{ }^{2} / N_{j}\right)^{1 / 2}}
$$

where $e_{i j}$ is the uncertainty for the flux $f_{i j}$ at the pixel $i$ and $N_{j}$ is the number of pixels within the above wavelength windows intervening in the summation. Spectra with $\mathrm{S} / \mathrm{N}<1$ are discarded from further calculations. This threshold eliminates around $22 \%$ of the remaining spectra from the calculations. Finally, the mean $\mathrm{S} / \mathrm{N}$ values are used to assign a weight of the form

$$
w_{j}=\frac{1}{\mathrm{~S} / \mathrm{N}_{j}^{-2}+\sigma^{2}}
$$

to each quasar spectrum, where $\sigma=0.1$ limits the potential excessive contribution from spectra with very high $\mathrm{S} / \mathrm{N}$. In Mas-Ribas et al. (2017) we tested that small variations around this value do not alter our results, and we therefore use the same quantity. The final mean quasar spectrum is then computed as a weighted mean using the aforementioned weight values.

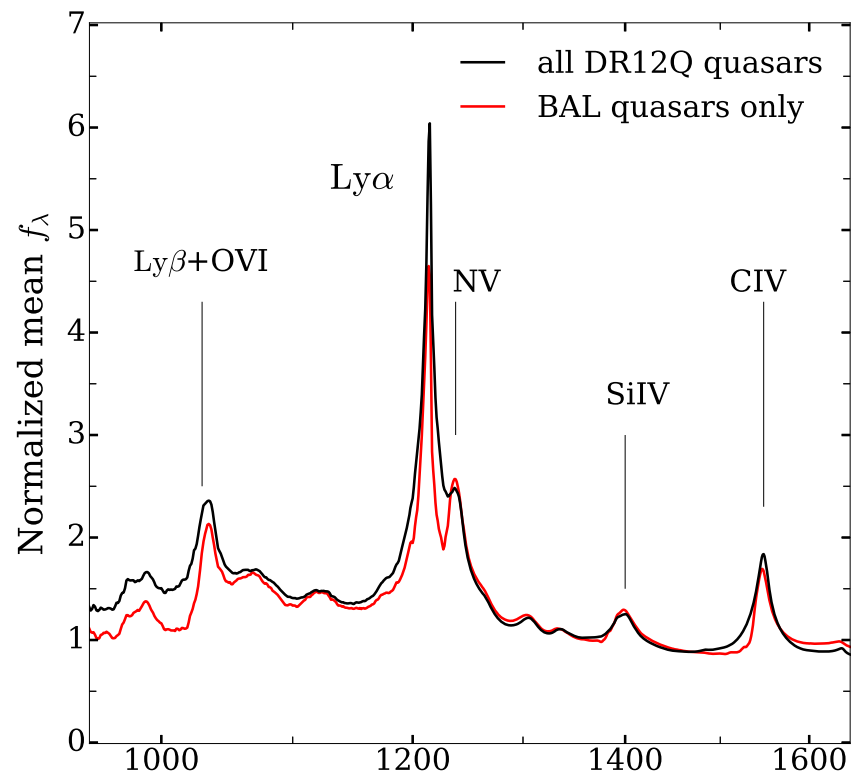

Figure 3. Mean quasar spectrum considering all DR12Q quasars (black line) and only those containing broad absorption troughs (red line). The average outflow absorption features of the elements with highest opacities, such as $\mathrm{C}$ IV, $\mathrm{N} \mathrm{V}, \mathrm{Ly} \alpha$, and $\mathrm{O} \mathrm{VI}$, are visible in the left part of the respective emission lines in the red spectrum. The horizontal axis is logarithmically spaced for visualization.

Figure 3 shows the difference between the spectrum considering all the DR12Q quasar spectra (black line), and only those containing troughs (red line). A broad absorption trough is visible to the left of the emission lines of species with the highest opacities, i.e., $\mathrm{CIV}, \mathrm{N}$ V, Ly $\alpha$, and $\mathrm{O}$ VI, in the trough-only spectrum. The use of this biased mean spectrum would result in the underestimation of the true line equivalent widths. We have checked that including the quasars containing troughs in the total quasar sample for this calculation does not significantly affect the resulting mean quasar spectrum. The relative difference between the mean quasar built from the whole quasar sample and the quasar built only from quasars that do not contain outflow troughs is $\lesssim 6 \%$ at the peaks of the quasar emission lines. In the flatter quasar regions where the absorption troughs are found, the differences are $\lesssim 2 \%$. A similar mean spectrum, except for the lack of corrections on the average absorption of the $\operatorname{Ly} \alpha$ forest, is illustrated in Figure 3 of Mas-Ribas et al. (2017), and a movie showing the build-up of the mean quasar spectrum with an increasing number of spectra is publicly available at https://github.com/lluism/ DLA_movies.

We have tested the impact on our results of using an individual mean spectrum for every quasar spectrum, derived from the spectra of its nearest neighbors, instead of our mean quasar calculation. In the nearest-neighbor approach, the mean spectrum for each quasar containing troughs is obtained by computing the mean spectrum of its 40 trough-free nearest neighbors in the entire DR12Q catalog. The proximity between the spectra is defined as the Euclidean distance in normalized flux units as

$$
d_{j k}=\sqrt{\sum_{i}\left(f_{i, j}^{N}-f_{i, k}^{N}\right)^{2}},
$$

where $i$ are the pixels in the quasars $j$ and $k$, and $f_{i}^{N}$ is the flux once normalized by the average flux in the wavelength 
windows $1300<\lambda_{\mathrm{r}} / \AA<1383$ and $1408<\lambda_{\mathrm{r}} / \AA<1500$. We have considered only the spectral regions above $1280 \AA$ in the quasar rest frame to avoid the impact of the strongest quasar emission lines (Davies et al. 2018). When we use the mean spectra from the nearest-neighbor approach, we obtain outflow composite spectra that are slightly less noisy redward of the C IV absorption doublet than those that we obtain when we use the mean of all the quasars. This is because the individual mean, i.e., from the nearest neighbors, matches the quasar spectra better than the overall mean. However, the noise for the nearest-neighbor case is significantly higher at short wavelengths, especially in the Ly $\alpha$ forest, because not all the spectra in the DR12Q catalog cover the region below $\sim 1500 \AA$ in the quasar rest frame. If one considers only the spectra that cover the entire forest region $(\gtrsim 912 \AA)$, then the number of spectra and candidate neighbors is reduced and the resulting outflow composite does not show a significant improvement compared to using our mean spectra. We therefore conclude that our mean quasar spectrum method is adequate for our calculations.

\subsection{Outflow Composite Spectra Calculation}

We compute the composite rest-frame outflow spectrum as a weighted mean, now only using the quasar spectra that contain troughs, and centering these spectra at the position of the minimum flux within the troughs as given in DR12QBAL. Perrotta et al. (2018) recently stacked outflow absorption features in high-resolution spectra, and they found that centering the spectra at the position of the deepest (minimum flux) C IV component yields the best alignment between C IV and the other absorption features, especially those of lowionization species. In our case, given the low resolution of the BOSS spectra, the position of the minimum flux can be determined with higher accuracy than the limits of the troughs or other points, which in turn results in more precise (narrower) absorption profiles. Indeed, our stacks do not resolve the two lines of the C IV doublet and show much broader absorption features when we center the spectra at the centroid of the troughs or at the minimum or maximum trough limits. This test suggests that the minimum-flux position mostly has a physical origin and that the effect of noise is small. We elaborate further on the impact of choosing the minimum-flux position on our results in Section 6.1.

The redshift of the troughs is computed using the velocity offset from the quasar (i.e., from the C IV quasar emission lines) given in the DR12QBAL catalog, and the expression

$$
\frac{v}{c}=\frac{\left(1+z_{\mathrm{q}}\right)^{2}-\left(1+z_{\mathrm{t}}\right)^{2}}{\left(1+z_{\mathrm{q}}\right)^{2}+\left(1+z_{\mathrm{t}}\right)^{2}} .
$$

Here $v$ and $c$ are the trough position and the speed of light, respectively, and $z_{\mathrm{t}}$ and $z_{\mathrm{q}}$ denote the redshifts of the trough and the quasar, respectively. We then rebin each outflow spectrum into an evenly spaced array of pixel width $d \lambda=0.3 \AA$, similar to the maximum BOSS pixel resolution $\left(\simeq 69 \mathrm{~km} \mathrm{~s}^{-1}\right.$ in velocity space), and normalize them with the corresponding normalization factor obtained in the mean spectrum calculation. Next, the normalized spectra are divided by the mean quasar spectrum, shifted and rebinned to the new outflow wavelength array, to eliminate the quasar footprint and obtain the individual outflow absorption spectra. The final outflow composite spectrum results from computing the weighted mean of these individual outflow absorption spectra, where the weighting factors are the weights obtained for every spectrum in the mean quasar calculation. At the end of these calculations, the composite spectra present a residual offset of $\approx 200 \mathrm{~km}^{-1}$ from the theoretical position expected for the absorption lines. This is because the position of the absorption trough is measured from the peak of the $\mathrm{C}$ IV quasar emission line, which is generally found at a rest-frame wavelength of $\approx 1549.4 \AA$, while the minimum flux within the trough is denoted by the blue line of the $\mathrm{C}$ IV doublet at $\approx 1548.2 \AA$, thus separated from the emission peak by $\approx 250 \mathrm{~km}^{-1}$. This separation is consistent with the observed offset considering our flux pixel resolution of $\sim 69 \mathrm{~km} \mathrm{~s}^{-1}$. We simply correct for this offset by shifting our composite spectra to the theoretical position of the absorption lines.

We build 36 outflow composite spectra drawn from subsamples of the overall trough catalog, all computed as described above. We divide the outflow velocity range into nine bins and consider two additional subsamples for low velocities. The trough width range is divided into five samples and the trough minimum velocity into seven. The quasar redshift and magnitude ranges are divided into six bins each. The choice for the subsamples and their number is performed with the aim to divide the data into enough bins to study the dependences on outflow and quasar parameters in our companion work, while containing a similar number of troughs in each bin to reach similar $\mathrm{S} / \mathrm{N}$ levels. When effects are observed at specific values (i.e., at low outflow velocities), we create additional bins to study them in more detail, even though this reduces the number of troughs. Together with the stack of all troughs in the catalog, these subsamples are detailed in Table 2. The first column lists the selection criteria to build each subsample, where trough min. velocity is the minimum velocity of each trough, and the bottom group is parameterized using the absolute $i$-band magnitude at $z=2$ of the quasars, as reported in DR12Q (Pâris et al. 2017). The second column in Table 2 quotes the mean value for each selection parameter, and the third column lists the values of the intercept that we used for the calculation of the $a$ parameters in the absorption profile (Equation (5) and Section 3.3.3). The fourth column denotes the number of spectra considered for the composite of each subsample. We publicly release our composite spectra at https://github.com/lluism/BALs, and their analysis is performed in our companion paper, Mas-Ribas (2019).

\subsection{Line Profile Fitting}

We describe the wavelength ranges considered for the modeling of the absorption lines and the continua in Section 3.3.1, the formula for the absorption profile fitting in Section 3.3.2, and the computation of the absorption width in Section 3.3.3. For the modeling of the absorption lines we consider the set of atomic transitions listed in Table 1, although not all of them are detected in all our stacks.

\subsubsection{Line Windows and Local Continua}

The first step toward obtaining a reliable fit to the absorption lines is to renormalize the outflow composite spectra. This modification is required because the impact of neighboring 
Table 2

Outflow Samples

\begin{tabular}{|c|c|c|c|}
\hline $\begin{array}{l}\text { Selection Criteria } \\
\text { All }\end{array}$ & Mean & $\begin{array}{c}n(\AA)^{\mathrm{a}} \\
0.437 \pm 0.029\end{array}$ & $\begin{array}{c}\text { No. Troughs } \\
59872\end{array}$ \\
\hline \multicolumn{4}{|c|}{ Outflow Velocity $\left(\mathrm{km} \mathrm{s}^{-1}\right)$} \\
\hline$v<200$ & 139 & $0.335 \pm 0.064$ & 185 \\
\hline$v<350$ & 264 & $0.331 \pm 0.045$ & 3167 \\
\hline$v<650$ & 315 & $0.254 \pm 0.040$ & 4355 \\
\hline $350 \leqslant v<650$ & 452 & $0.278 \pm 0.038$ & 1188 \\
\hline $650 \leqslant v<1500$ & 1122 & $0.291 \pm 0.037$ & 2278 \\
\hline $1500 \leqslant v<3000$ & 2233 & $0.387 \pm 0.081$ & 6706 \\
\hline $3000 \leqslant v<5000$ & 3980 & $0.444 \pm 0.095$ & 7727 \\
\hline $5000 \leqslant v<8000$ & 6426 & $0.462 \pm 0.031$ & 10,183 \\
\hline $8000 \leqslant v<13,000$ & 10,330 & $0.439 \pm 0.050$ & 12,488 \\
\hline $13,000 \leqslant v<17,500$ & 15,208 & $0.354 \pm 0.078$ & 9075 \\
\hline$v \geqslant 17,500$ & 20,867 & $0.302 \pm 0.138$ & 10,737 \\
\hline \multicolumn{4}{|c|}{ Trough Width $\left(\mathrm{km} \mathrm{s}^{-1}\right)$} \\
\hline$v<560$ & 508 & $0.227 \pm 0.045$ & 11,250 \\
\hline $560 \leqslant v<708$ & 629 & $0.264 \pm 0.094$ & 8420 \\
\hline $708 \leqslant v<1260$ & 951 & $0.305 \pm 0.149$ & 13,488 \\
\hline $1260 \leqslant v<2240$ & 1704 & $0.381 \pm 0.057$ & 10,266 \\
\hline$v \geqslant 2240$ & 5179 & $0.560 \pm 0.056$ & 20,125 \\
\hline \multicolumn{4}{|c|}{ Trough Minimum Velocity $\left(\mathrm{km} \mathrm{s}^{-1}\right)$} \\
\hline$v<300$ & 128 & $0.423 \pm 0.048$ & 2574 \\
\hline $300 \leqslant v<2300$ & 1236 & $0.431 \pm 0.075$ & 10,713 \\
\hline $2300 \leqslant v<5300$ & 3694 & $0.381 \pm 0.132$ & 10,661 \\
\hline $5300 \leqslant v<9000$ & 7113 & $0.352 \pm 0.032$ & 10,356 \\
\hline $9000 \leqslant v<13,500$ & 11,183 & $0.400 \pm 0.082$ & 10,570 \\
\hline $13,500 \leqslant v<18,500$ & 15,953 & $0.409 \pm 0.054$ & 10,059 \\
\hline$v \geqslant 18,500$ & 21,186 & $0.463 \pm 0.089$ & 8630 \\
\hline \multicolumn{4}{|c|}{ Quasar Redshift } \\
\hline$z<1.95$ & 1.77 & $0.449 \pm 0.093$ & 10,580 \\
\hline $1.95 \leqslant z<2.20$ & 2.09 & $0.455 \pm 0.047$ & 10,584 \\
\hline $2.20 \leqslant z<2.37$ & 2.28 & $0.455 \pm 0.042$ & 10,812 \\
\hline $2.37 \leqslant z<2.60$ & 2.48 & $0.414 \pm 0.066$ & 10,591 \\
\hline $2.60 \leqslant z<3.00$ & 2.79 & $0.452 \pm 0.009$ & 10,880 \\
\hline$z \geqslant 3.00$ & 3.38 & $0.461 \pm 0.159$ & 10,098 \\
\hline \multicolumn{4}{|c|}{ Quasar Magnitude (mag) } \\
\hline$-25.0 \leqslant M_{i}<-22.0$ & -24.6 & $0.522 \pm 0.191$ & 10,972 \\
\hline$-25.5 \leqslant M_{i}<-25.0$ & -25.3 & $0.482 \pm 0.100$ & 10,912 \\
\hline$-26.0 \leqslant M_{i}<-25.5$ & -25.8 & $0.428 \pm 0.061$ & 12,932 \\
\hline$-26.5 \leqslant M_{i}<-26.0$ & -26.2 & $0.415 \pm 0.040$ & 12,020 \\
\hline$-27.0 \leqslant M_{i}<-26.5$ & -26.7 & $0.387 \pm 0.037$ & 8949 \\
\hline$-30.0 \leqslant M_{i}<-27.0$ & -27.5 & $0.383 \pm 0.074$ & 7749 \\
\hline
\end{tabular}

Note.

a This parameter denotes the values of the intercept in Equation (6) and is an indicator of the width of the absorption lines in the composite spectra.

absorption features and/or the Ly $\alpha$ forest in some cases results in absorption-free spectral regions that deviate significantly from the unity transmission value in the final spectra. We renormalize each outflow composite spectrum by dividing it by a pseudo-continuum. This is computed via smoothing the composites with a Gaussian kernel of width $\sigma_{\mathrm{G}}=10$ pixels, showing no substantial differences for other values within the range $5 \leqslant \sigma_{\mathrm{G}} \leqslant 15$. For the smoothing, we disregard the wavelength ranges $-1.7 \leqslant\left(\lambda-\lambda_{\mathrm{c}}\right) / \AA \leqslant 1.0$ around the absorption lines, where $\lambda_{\mathrm{c}}$ denotes the line center. The ranges are asymmetrically centered around the lines to account for the presence of the line-locked component in the low-wavelength side of each absorption feature. We note that this renormalization washes out the broad absorption wings that sometimes extend blueward of the strongest absorption transitions (most notable for C IV), and thus mostly retains the central and deep regions where the absorption lines are defined. In turn, the equivalent widths of some of the strongest lines may be underestimated and should not be adopted without further considerations. Despite this renormalization, the final absorption spectrum is still not completely flat around the absorption lines in some cases. For this reason, we use a local continuum over the absorption lines for the profile fitting as described below.

The absorption profiles are modeled in a wavelength window with a half-width of $4 \AA$ on each side of the line center, plus an additional extent of $500 \mathrm{~km} \mathrm{~s}^{-1}$ on the low-wavelength limit to account for the presence of the line-locking component. This window size is large enough to cover the lines and their linelocked components in all our composite spectra. When two or more line windows overlap, we consider a single window spanning from the lowest to the highest limit of the intervening windows, and the profiles of all the lines within this range are modeled at the same time. This approach results in the simultaneous modeling and measurement of up to five absorption lines in general. In two cases we obtain a window containing 10 lines: in the wavelength range around $\sim 1125 \AA$, and in the wavelength range around $\sim 1195 \AA$. We simply divide both ranges into two parts containing five lines each and treat them as separate features. Most lines in these ranges are weak, and their precise measurement does not impact our findings, so we do not attempt more complex computations.

The local continuum over each absorption feature is computed by fitting a linear regression to the flux pixels next to the absorption window. We consider the outflow transmission pixels beyond the limits of the line (lines) window up to a distance of $9 \AA$ on both sides from the line center (or the minimum and maximum line centers for the case of multiple lines). We require a minimum total number of 15 pixels in these ranges. This value is chosen to reduce the impact of spurious flux pixels while representing the regions near the absorption lines. If the total number of pixels in the two ranges is smaller because pixels are not considered as they belong to neighboring line windows or are affected by skylines, both ranges are recursively enlarged by $0.4 \AA$ until the minimum number is reached.

\subsubsection{Profile Estimator}

The modeling of the absorption lines considering a simple feature clearly does not reproduce the observed profiles. The presence of the potential line-locking component in our outflow spectra requires for every absorption line the joint fit of two features: the line itself, and the line-locked counterpart. For clarity, we refer to these two components as line and linelocking from here onward, and we account for the two contributions in all our measurements.

We fix the center of the line-locking considering that $v_{\mathrm{c}}-v_{11} \equiv \Delta_{v}=-497 \mathrm{~km} \mathrm{~s}^{-1}$, where $v_{\mathrm{c}}$ and $v_{11}$ are the positions of the line and line-locking in velocity space, respectively, and the value corresponds to the separation between the two lines of the C IV $\lambda \lambda 1548,1550$ doublet. We choose this value because the stacking of C IV troughs results in the enhancement of the C IV line-locking feature, while it dissipates the contribution from other doublets. We have tested that variations of $\leqslant 15-20 \mathrm{~km} \mathrm{~s}^{-1}$ around the nominal C IV 
separation value (corresponding to $\sim 22 \%-29 \%$ of the pixel size) generally do not produce significant differences in the fits. We also require that the ratio between the line and line-locking components (i.e., $d / b$ below) remain the same when the lines that are being fit belong to the same atomic doublet.

We perform a least-squares fit to each line and line-locking pair assuming a profile of the form

$$
\begin{aligned}
F_{\lambda}= & C_{\lambda} \exp \left[-b \exp \left(\frac{-\left(\lambda-\lambda_{\mathrm{c}}\right)^{2}}{2 a^{2}}\right)\right. \\
& \left.-d \exp \left(\frac{-\left(\lambda-\lambda_{\mathrm{ll}}\right)^{2}}{2 a^{2}}\right)\right],
\end{aligned}
$$

where the position of the line-locking in wavelength space is obtained with the relation $\lambda_{11}=\lambda_{\mathrm{c}}\left(1+\Delta_{v} / c\right)$, noting the negative sign of $\Delta_{v}$. The parameter $C_{\lambda}$ in Equation 5 represents the local continuum at a wavelength $\lambda$ in the renormalized restframe outflow composite spectrum, and $b, d$, and $a$ are free parameters. The $a$ parameter is set to be the same for the line and line-locking, which yields a substantial improvement of the fits, especially when multiple features are fit at the same time and degeneracies between them can occur. Furthermore, we do not observe significant differences between the paired $a$ parameters when they are allowed to be different. However, the $a$ parameter is related to the width of the absorption features, which is mostly driven by the spectrograph resolution in the BOSS spectra, except for the broadest features. Because of this dependence on resolution, differences between the widths of the line and line-locking that are not detectable in our spectra can exist. It is important that future (higher resolution) analyses revisit these values because the line width carries relevant information on the kinematic properties of the media.

\subsubsection{Fixing the Line Widths}

In practice, we fix the value of the parameter $a$ in our calculations, taking into account its dependence on the spectrograph resolution mentioned above and the evolution of the latter with wavelength. In other words, instead of using the $a$ parameters resulting from the profile fit that show a large dispersion, we use these fitted values to build a relation with wavelength that will yield the value of $a$ at any wavelength. This approach yields a reduction in the number of free parameters and in turn an improvement of the fits to the absorption profiles because the model is less sensitive to the effect of noise and other contaminants.

We first parameterize the evolution with wavelength in 34 of our outflow composite spectra to cover a broad range of trough parameter values and to assess possible dependences of the evolution on these parameters. We consider the total sample, the 6 spectra for the subsamples in outflow redshift, the 6 for the trough width, 4 for the low-velocity limit of troughs, the 6 for quasar magnitudes, and the 11 for the outflow velocities, all of them detailed in Table 2. We fit all the features in Table 1 for each of these spectra, with the $a$ parameter for every line and line-locking pair allowed to be free, and keep the $a$ values when the fit exists (we consider that a fit does not exist and ignore its outcome when it returns unphysical, i.e., null or negative, values for some of the parameters). An increase in the $a$ parameter with wavelength is apparent in all cases. However, a large scatter in the individual $a$ values, partially driven by

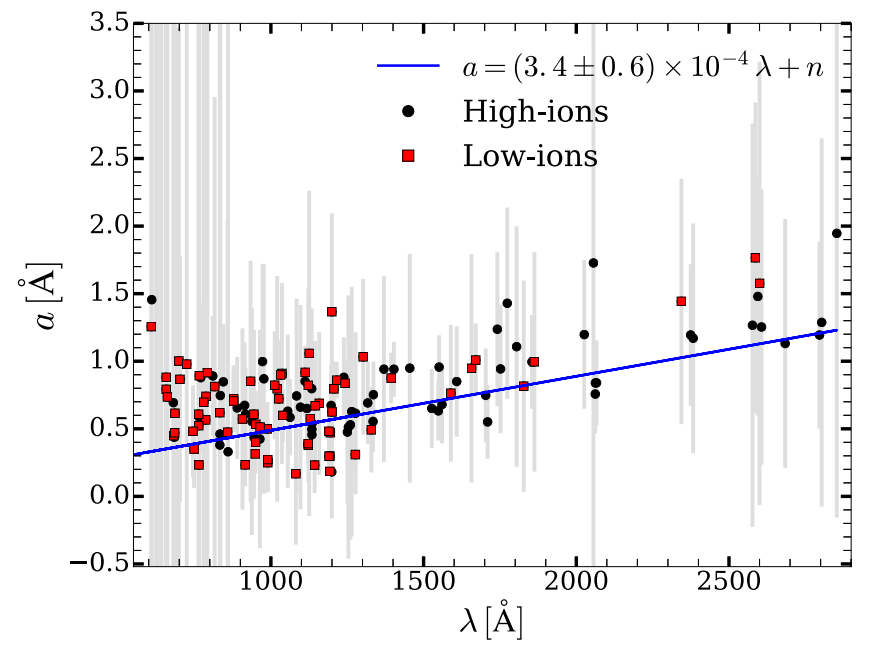

Figure 4. Mean values of the $a$ parameters with wavelength, computed from the 34 composite outflow spectra detailed in the main text. There are no apparent differences between the trends for low- (red squares) and highionization (black dots) species, and a general increase in $a$ with wavelength is visible, but with a large scatter (gray lines). The blue line denotes the linear fit that yields the slope value $m=3.4 \times 10^{-4}$, which we used to obtain the $a$ parameter in the individual stacks.

degeneracies between blended absorbers and by high noise in the spectra of some stacks that especially affects the weakest features, does not enable us to constrain the wavelength dependence with accuracy in the individual stacks. Thus, we decide to parameterize the slope of the evolution with wavelength using the mean of the $a$ values from the 34 subsamples, which is consistent with the lack of visible differences in the evolutions between subsamples or between low- and high-ionization species. We finally fit a linear regression to these mean values considering the still large error bars that arise from the dispersion of values around the mean and use its slope of $m=3.4 \times 10^{-4}$ for the computation of the $a$ parameter in all the composite subsamples. This slope is similar to the slope we obtained for DLAs in Mas-Ribas et al. (2017) $\left(2.3 \times 10^{-4}\right)$, indicating that the evolution is mostly driven by the values of the wavelength and not by physical effects specific to outflow environments.

Figure 4 displays the mean value of the $a$ parameters with wavelength, computed using the 34 composite spectra of the subsamples described above. A general increase in $a$ with wavelength is visible, but with significant scatter (error bars denoted by gray lines), and there are no apparent differences between low- (red squares) and high-ionization (black dots) species. The blue line denotes the linear fit to the data with their uncertainty, which yields the slope value of $m=3.4 \times 10^{-4}$.

With the value of the slope now fixed, we again apply a linear regression to the $a$ values of each subsample to obtain the individual best-fit value of the intercept, $n$. In this case, we only consider the $a$ parameters of the strong transitions H I $\lambda 1215$, NV $\lambda 1238, \quad$ NV $\lambda 1242, \quad$ Si IV $\lambda 1393, \quad$ Si IV $\lambda 1402, \quad$ and C IV $\lambda 1548$, where the $a$ parameters are measured with the highest precision. In all cases we visually inspect the fits to these absorption features and discard those that appear dubious or affected by contaminants. The best-fit values of the $n$ parameters for each subsample are reported in Table 2. Finally, the value of the $a$ parameter for an absorption feature at 
wavelength $\lambda$ can be obtained as

$$
a=(3.4 \pm 0.6) \times 10^{-4} \lambda+n
$$

where $n$ takes on the values in Table 2 for each spectrum, and the parameters are in units of Ångstrom.

\section{Dissection of an Outflow Composite Spectrum}

Figure 5 displays the outflow composite spectrum resulting from the subsample of troughs with outflow velocities within the range $1500 \leqslant v<3000 \mathrm{~km} \mathrm{~s}^{-1}$. The light blue color represents the transmitted flux regions, and dark blue highlights the absorbed regions where the transmission falls below the $1 \sigma$ noise, shown in orange. This noise is computed for each pixel as the median of the variance of the flux around the unity level and considering a region centered at the pixel with a halfwindow size of $25 \AA$. The pixels in regions corresponding to absorption lines (Section 3.3.1) are discarded for the noise calculation. Note that the scales of the axes for each range are set independently for visualization purposes.

This spectrum was renormalized using a pseudo-continuum as described in Section 3.3.1, but the local continua around the absorption features we used for the calculation of the equivalent widths were not applied because they depend on each feature. Regions with transmission above unity, visible on the right-hand side of the absorption features of O I $\lambda 1039$, $\mathrm{HI} \lambda$ 1215, NV $\lambda$ 1242, and $\operatorname{CIV} \lambda$ 1550, appear due to differences between the mean quasar spectrum we used for the normalization of the composites and the individual quasar spectra. In general, this flux excess indicates that the mean quasar spectrum presents weaker emission lines than those in the individual quasars. However, the effect of weighting the spectra also causes this effect because the higher $S / N$ is typically related to the brighter quasars, which in turn have weaker emission lines due to the Baldwin effect (Baldwin 1977; see Figure 3 in Mas-Ribas et al. 2017 and the associated text for details). We do not apply further corrections because this effect does not impact our results concerning the line-locking, and the equivalent widths of the lines are well fit given the fix value of their widths.

We have labeled the strong atomic transitions from Table 1. A number of them can be identified deep into the Ly $\alpha$ forest region. The strongest features are the well-resolved doublets of the high-ionization species S VI $\lambda \lambda 933,944$, O VI $\lambda \lambda 1031,1037$, N V $\lambda \lambda 1238,1242$, and C IV $\lambda \lambda 1548,1550$. These ions represent the typical effect from the strong radiation field that affects the outflow in general. However, other transitions of high- and low-ionization species are clearly visible, such as the first five hydrogen Lyman series lines from Ly $\alpha$ down to Lymanepsilon, i.e., H I $\lambda$ 937, and C III $\lambda$ 977, N III $\lambda$ 989, Si III $\lambda$ 1206, and the doublets PV $\lambda \lambda 1117,1128$, Si IV $\lambda \lambda 1393,1402$, AlIII $\lambda \lambda 1854,1862$, and $\mathrm{Mg}$ II $\lambda \lambda 2796,2803$. We have also labeled transitions of the low-ionization species Ni II, Fe II, O I, Si II, and C II. We find no evidence for the presence of molecular $\mathrm{H}_{2}$ features, although the line-locking component on the left-hand side of the absorption lines may overlap and mask the molecular hydrogen signatures. We study the physical properties of the outflows by analyzing this and the other composite spectra in our companion paper, Mas-Ribas (2019).

An absorption feature on the low-wavelength side of all lines, which is blended with the lines, is clearly visible for the case of the strongest transitions in Figure 5. We identify this feature as the CIV line-locking component of the corresponding lines and analyze it in more detail below.

\section{Line-locking}

We analyze the characteristics and nature of the line-locking components below. In Section 5.1 we discuss the C IV-driven features visible in our spectra, and search for signatures of $\mathrm{O}$ VI and $\mathrm{N}$ V in Section 5.2.

\subsection{Line-locking}

Figure 6 illustrates the fits to three of the strongest absorption features in the spectrum of Figure 5. From left to right, the panels cover the approximate wavelength ranges 1020-1040 А,

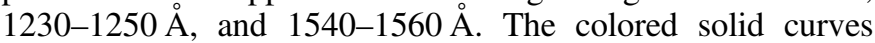
denote the fit to the individual lines, and the dashed curves are the fits to the corresponding line-lockings, following the same color code. The red curves represent the total fit in each panel, and the local continua are visible as straight lines above the features, where the colors simply result from the superposition of the individual curves and have no meaning. The C IV linelocking components at $497 \mathrm{~km} \mathrm{~s}^{-1}$ from the corresponding lines are clearly visible. This occurs for any absorption feature that is visible beyond the noise in all our composite spectra, regardless of the species or the ionization state, as expected from the theory of radiative acceleration in quasar outflows (e.g., Arav \& Begelman 1994; Arav \& Li 1994).

The leftmost panel in Figure 6 shows regions between the absorption lines that are not fit by our model. This is because we have selected the strongest lines in this region to illustrate the presence of line-locking, but we have not considered a number of weaker transitions that inhabit the regions in between, which sometimes overlap with the strongest lines. Our simple methodology does not yield reliable fits in cases of many strongly blended lines. The two strongest transitions we omitted are C II $\lambda 1036$ and the excited-state line C II $\lambda 1037$. However, the effect of these two lines together should be small, similar to the signal observed for C II $\lambda 1334$ and C II $\lambda 1335$ in Figure 5, because the oscillator strengths for all these lines are almost the same (Table 1). Other O I transitions also impact these regions, with strengths about half of the strength of $\mathrm{O} I \lambda$ 1302 in Figure 5, according to the relation of oscillator strengths. The three strongest lines that we consider for the fit are sufficient in many of the 36 stacks to produce a complete fit (see figures in the Appendix). In cases of blending or weak absorption features, the fit is sometimes better without the linelocking (or line), but we attribute this result to degeneracies between overlapping transitions or to the effect of noise, respectively, and not to physical causes. Some equivalent widths can be contaminated by these effects and yield unreliable measurements. Relevant (strong) transitions that are potentially affected by degeneracies are H I $\lambda 949$, H I $\lambda 1025$, O VI $\lambda 1037$, and the C IV $\lambda \lambda 1548,1550$ doublet. Instead, the Ly $\alpha$ transition, i.e., H I $\lambda 1215$, and the doublets N V $\lambda \lambda 1238,1242$ and Si IV $\lambda \lambda 1393,1402$ are well measured in most of our composite spectra.

The line-depth ratios between the line-locking and line features of Figure 6 vary broadly between $\sim 0.3$ and $\sim 0.8$, and the scatter increases when more lines are considered. This result suggests that line-locking occurs in a fraction of quasar outflows, the exact number depending on the uncertain value of the ratio, which can also be (partially) driven by differences between the 

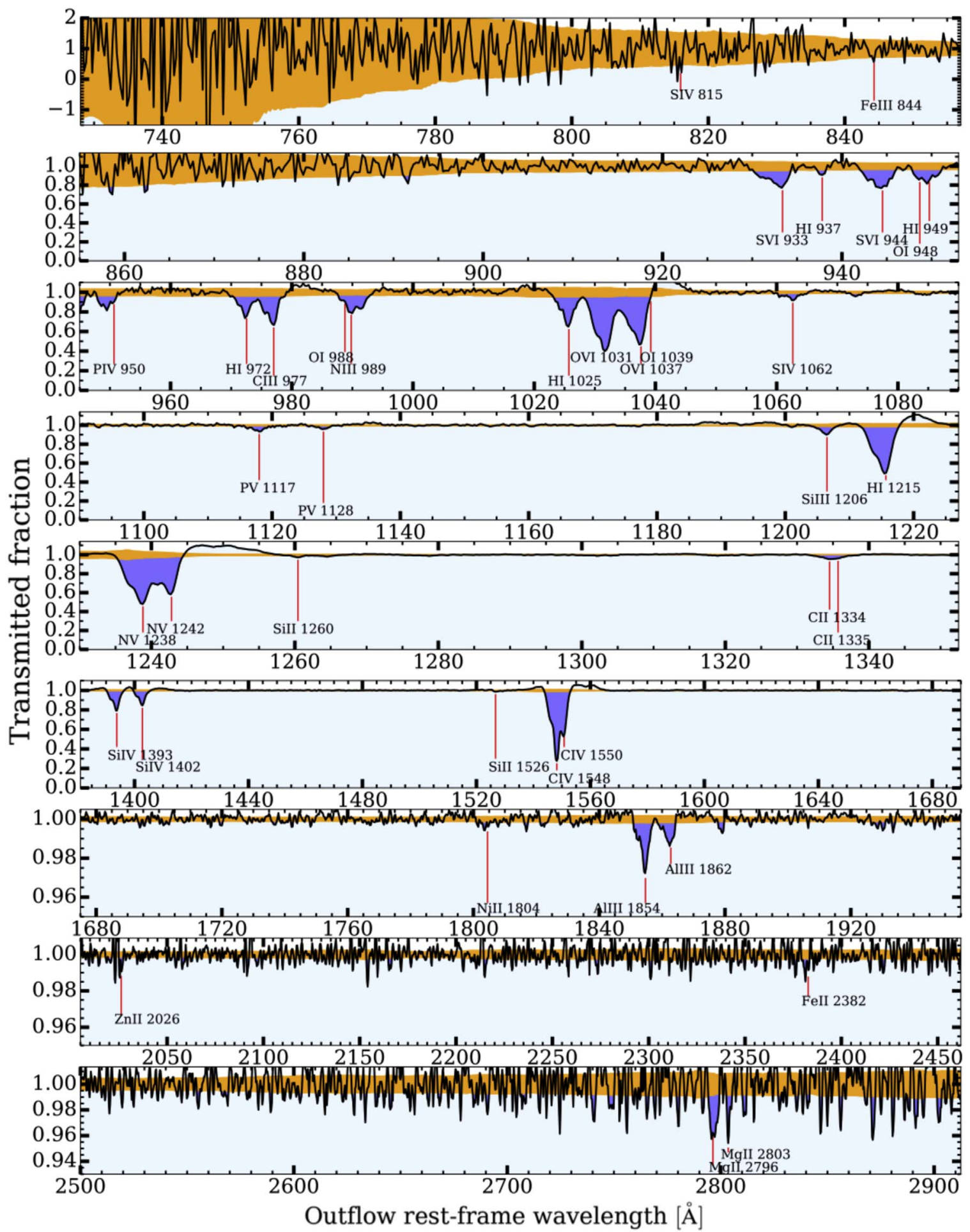

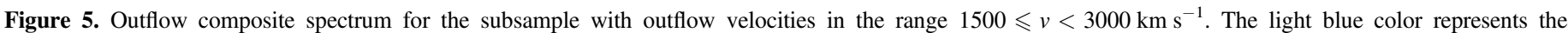

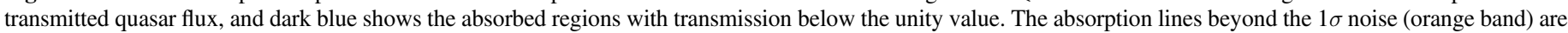

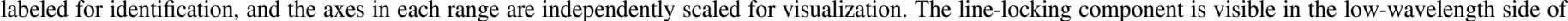

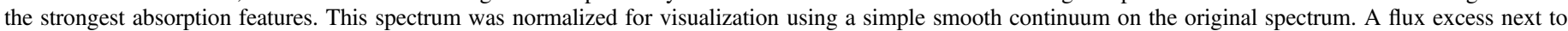

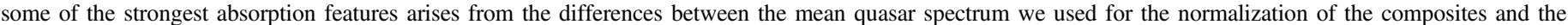
individual quasar spectra.

optical depths of the line and the line-locking. From the composite spectra of about 19,500 narrow $\left(<200 \mathrm{~km} \mathrm{~s}^{-1}\right) \mathrm{C}$ IV absorbers in SDSS quasars showing BALs, Bowler et al. (2014) inferred that $50 \%-75 \%$ of C IV systems in outflows are linelocked, which broadly agrees with our ratio values.
We show in the Appendix the fits to the previous three wavelength ranges, as well as that covering the Ly $\alpha$ absorption line and line-locking, for all our composites. The line-locking component is distinguishable in most cases. There are some cases where a good fit cannot be obtained due to the effects of 

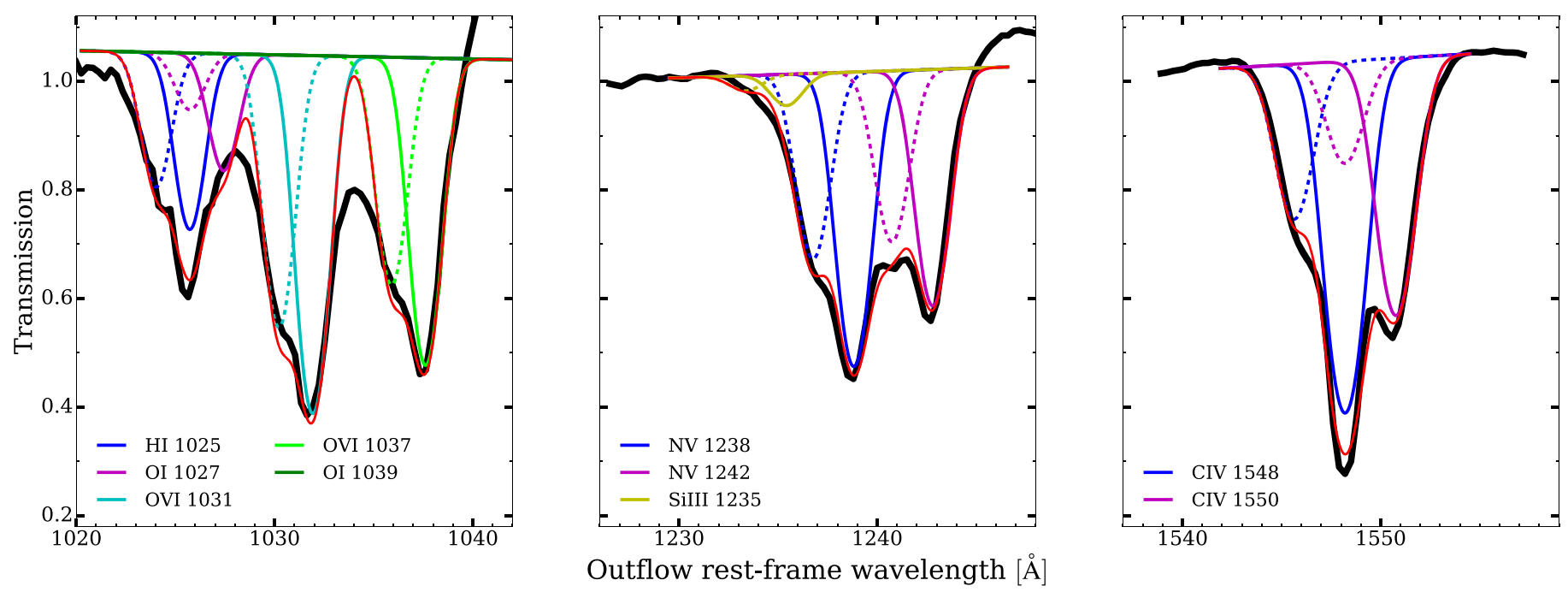

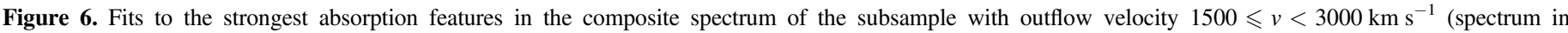

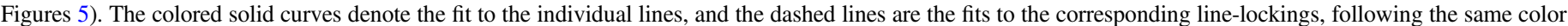

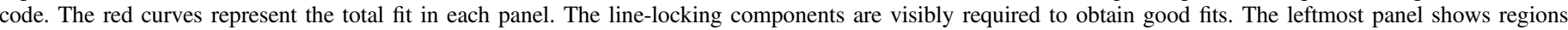

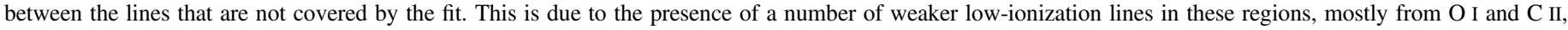
which are not included in our simple approach (see main text).
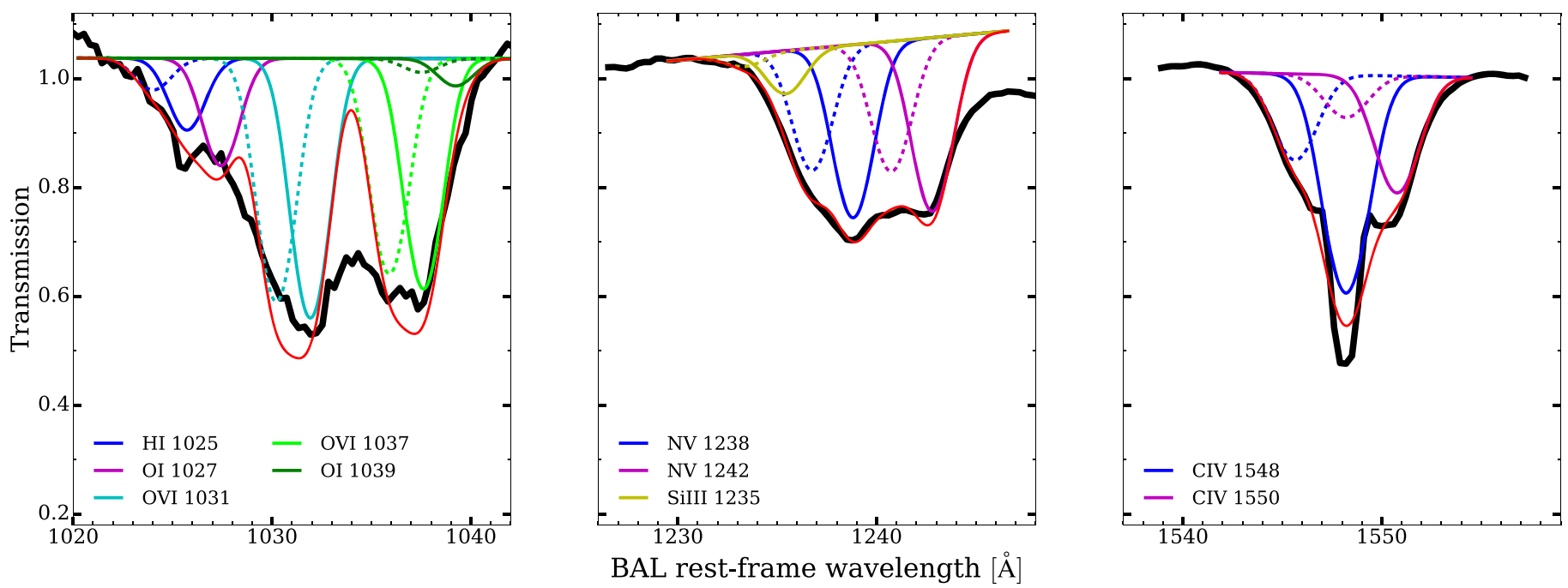

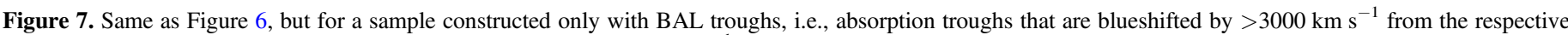
quasar emission line, and with a full width at half-minimum $\gtrsim 2000 \mathrm{~km} \mathrm{~s}^{-1}$.

noise or contaminants. These cases are left as blanks in the plots.

\subsubsection{Line-locking in BALs}

Our work aims at analyzing the quasar outflow properties based on the physical properties of the individual C IV absorption systems, and not on the nomenclature that is often assigned to the troughs themselves (e.g., BALs, mini-BALs, or NALs). We do so because these names may all refer to the same type of quasar outflows even though they account for different properties (Hamann et al. 2019; see also how these different terms can be accounted for considering the differences in outflow velocity and trough width in Mas-Ribas 2019). Because some of these terms are used very frequently, however, we briefly show here the presence of line-locking in an additional sample built from the strict definition of BAL systems. Specifically, BALs refer to absorption troughs that are blueshifted by $>3000 \mathrm{~km} \mathrm{~s}^{-1}$ from the respective quasar emission line, and with a full width at half-minimum $\gtrsim 2000 \mathrm{~km} \mathrm{~s}^{-1}$ (Weymann et al. 1991).

Figure 7 shows the fits to the same absorption features as in Figure 6 , but for the case of BALs. The presence of the linelocking components is again required to obtain good fits to the overall absorption features.

\section{2. $O V I$ and $N V$ Line-locking}

We search here for line-locking signatures of the O VI and $\mathrm{N}$ V doublets, which may have been washed out in our C IVtrough composite spectra. These ions may potentially participate in the radiative-acceleration process through line-locking given their high cosmic abundances and the similar strength of their absorption features compared to that of C IV (see, e.g., Figure 6 or the strong effects by these ions already suggested on the quasar spectrum of Figure 3). This argument is also supported by the results by Bowler et al. (2014), who modeled the photoionization state of quasar outflows and found that the 


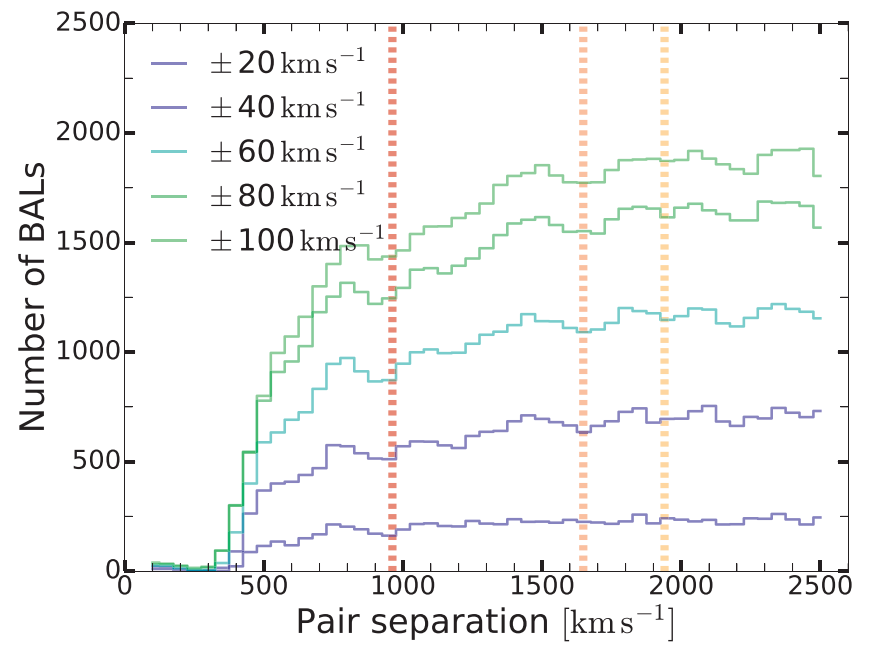

Figure 8. Number of pairs of C IV troughs separated by a given distance, from 100 to $2500 \mathrm{~km} \mathrm{~s}^{-1}$, in steps of $50 \mathrm{~km} \mathrm{~s}^{-1}$. We enable five offset values from the nominal positions of the troughs. The vertical dashed lines indicate the distances corresponding to the separations of the $\mathrm{NV}\left(962 \mathrm{~km} \mathrm{~s}^{-1}\right), \mathrm{O}$ VI $\left(1648 \mathrm{~km} \mathrm{~s}^{-1}\right)$, and Si IV (1940 $\left.\mathrm{km} \mathrm{s}^{-1}\right)$ doublets. No excess in the number of troughs is detected at the line-locking separations.

optical depths for O VI and N V are similar to that of C IV (their Figure 12). Furthermore, an additional line optical-depth autocorrelation analysis by the same authors confirmed the importance of these two ions for the overall opacity, although no absorption signatures were detected in their work.

As our absorber catalog only includes C IV systems and not other species, we cannot perform the same analysis to search for line-locking of other elements. Instead, we compute the number of pairs of C IV troughs in our catalog that are separated by a distance from 100 to $2500 \mathrm{~km} \mathrm{~s}^{-1}$ in steps of $50 \mathrm{~km} \mathrm{~s}^{-1}$. If linelocking from other species occurs with frequency and its signature is strong, the number of troughs at the locking separation should be larger than at other distances. Figure 8 shows the results of this calculation, where we have allowed for different offset values from the exact position of the expected troughs. The dashed vertical lines denote the separations of the $\mathrm{NV}$, O VI, and Si IV doublets. No excess in the number of troughs at the line-locking separations is detected in any case.

We conclude from this analysis that the $\mathrm{N} \mathrm{V}, \mathrm{Si} I V$, and O VI line-locking effects, if present, are not frequent and/or strong enough to produce an absorption feature detectable in the BOSS spectra.

\section{Discussion}

We discuss the reality of the C IV line-locking features in Section 6.1, the possible causes for the non-detection of linelocking from other species in Section 6.2, and compare with previous work in Section 6.3.

\subsection{Is It Really Line-locking?}

The presence of a C IV line-locking-like feature on the blue side of almost all the absorption lines is apparent in our stacks, but we found no conclusive evidence for the existence of the $\mathrm{O}$ VI, N v, or other line-locking effects. We revisit here the effects of our methodology that may compromise the reality of the C IV line-locking. We use the same composite spectrum as we analyzed above for consistency, the subsample with outflow velocity $1500 \leqslant v<3000 \mathrm{~km} \mathrm{~s}^{-1}$. We do not compute the additional $\sim 400$ stacks that arise from splitting each of the 36 spectra into the subgroups detailed below for testing all the composites. The impact from noise can be different for each composite, but considering the figures in the Appendix, it seems unlikely that noise can drive all the observations.

In Section 3.2 we checked that centering the outflow spectra at the position of the minimum flux within the absorption troughs yields the most refined (spectrally resolved) composite spectra. This result is consistent with the findings by Perrotta et al. (2018) and suggests that the origin of the minimum flux is mostly physical, i.e., due to the presence of the stronger absorption component among the absorption trough, and not driven by noise. In the case of one single C IV doublet, the blue (shorter wavelength) line is expected to be within one and two times stronger than the red line, the exact value set by atomic physics (i.e., by the ratio between the two oscillator strengths) and the level of saturation of the lines. In a noise-free doublet, the minimum flux will therefore always appear at the position of the blue line, except in the cases where the two lines are saturated and have the same minimum flux. Thus, although the minimum flux can be mostly governed by the lines, in cases of strong saturation, these minima can be identified at the position of the red line of the doublet, with the corresponding location of the blue line at the position of the expected line-locking component. If this occurs very frequently, a residual feature can be visible in the stacks. Furthermore, the effect of high noise levels could contribute to increase this problem.

We analyze the aforementioned potential impacts from the noise and strength of the absorption lines below. Figure 9 shows the comparison of composite spectra drawn from the trough subsample with outflow velocity $1500 \leqslant v<3000 \mathrm{~km} \mathrm{~s}^{-1}$, considering different $\mathrm{S} / \mathrm{N}$ thresholds (given for the whole spectrum in DR12Q; upper panel), and minimum-flux values (transmission) of the troughs (values from the DR12QBAL; lower panel). The left and right panels illustrate the regions blueward of the rest-frame absorption $\operatorname{Ly} \alpha$ line and the C IV doublet, respectively, and the dashed lines denote the position of the C IV line-locked components. The top panels show that the line-locking features remain almost constant when the $\mathrm{S} / \mathrm{N}$ of the spectra is increased, reflecting the small impact of the noise on our stacks. The line-locked features are also visible for the different ranges of transmission in the bottom panels, indicating the almost negligible effect from strong saturation that could increase the fraction of minimum-flux positions derived from the red line of the doublets. More important, the ratios between the lines and line-lockings remain almost the same in all cases, highlighting the relation between the two components driven by the line-locking effect.

We conclude that the absorption features detected blueward of the absorption lines in the deepest regions of the absorption features of our composite outflow spectra are the result of the C IV line-locking effect, which demonstrates the significant contribution from radiation to the acceleration of quasar outflows.

\subsection{On the Non-detection of $N V$ and $O V I$ Line-locking}

It is possible that physical effects do prevent line-locking from $\mathrm{N} \mathrm{V}$ and $\mathrm{O}$ VI to occur. Overionization of the medium inhabited by these species can suppress radiative acceleration from theses species because it results in too low opacities and optically thin 

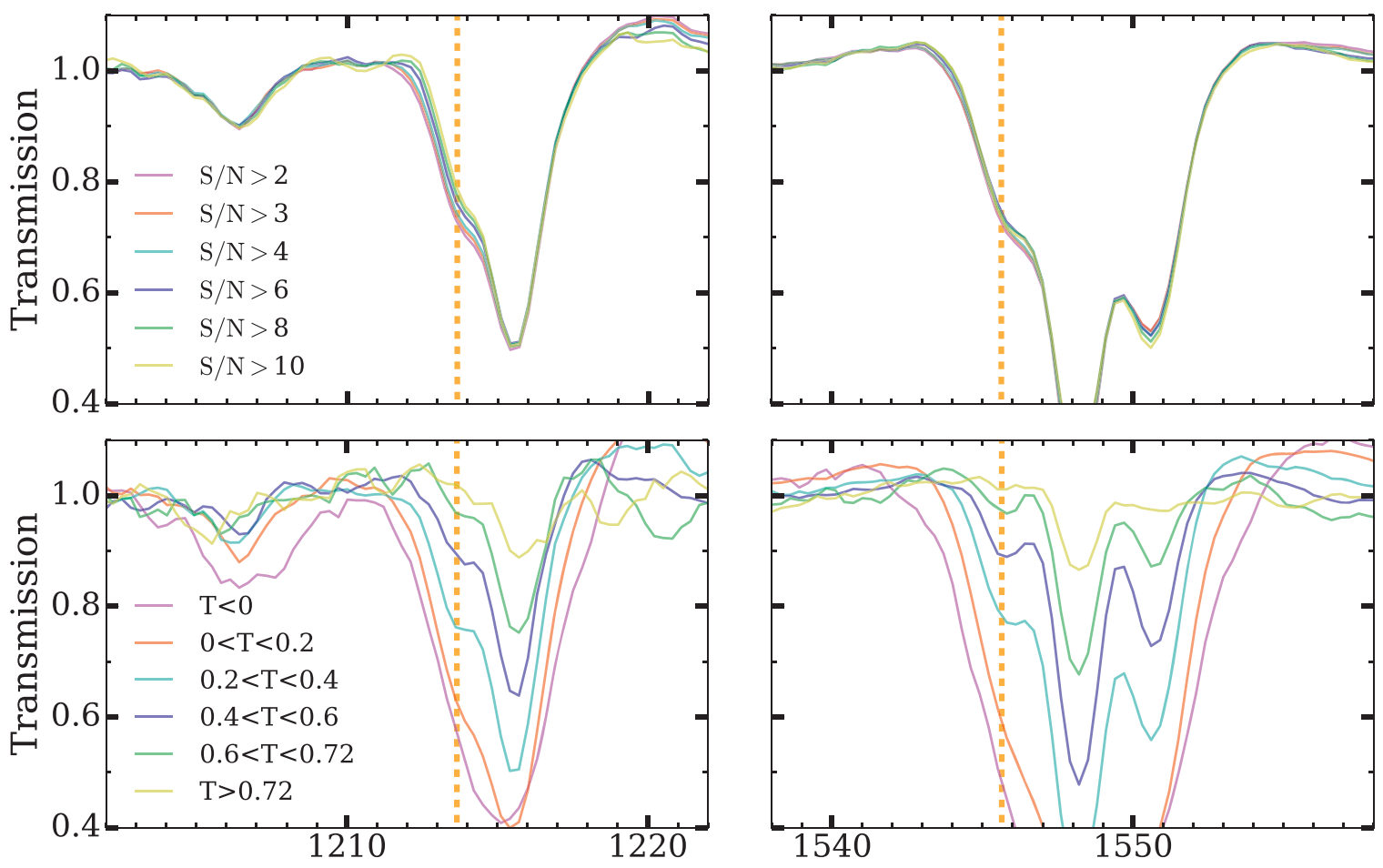

\section{BAL rest-frame wavelength $[\AA]$}

Figure 9. Comparison of composite spectra drawn from the trough subsample with an outflow velocity $1500 \leqslant v<3000 \mathrm{~km} \mathrm{~s}^{-1}$ and considering different $\mathrm{S} / \mathrm{N}$ thresholds (given for the whole spectrum in DR12Q; upper panel), and minimum-flux values (transmission) in the absorption troughs (values from the DR12QBAL; lower panel). The left and right panels illustrate the regions blueward of the rest-frame absorption Ly $\alpha$ and C IV doublet features, and the dashed lines denote the position of the C IV-locked components. The line-locking feature is visible in all cases, which suggests that it is real and not an artifact from the combination of doublets centered at different lines of the C IV doublet due to the noise or line strength.

media (Murray et al. 1995; Chelouche \& Netzer 2003; Leighly 2004; Proga \& Kallman 2004; Proga 2007a; Laor \& Davis 2014; Wang et al. 2015). However, C IV has a lower ionization potential and typically traces cooler and denser media than the oxygen and nitrogen ions. This region could also be shielded (by the highest ions themselves) from the high-energy radiation field and thus enable radiative acceleration from $\mathrm{C}$ IV line-locking to take place. We discuss the potential impact of the outflow properties and its structure on the presence of the $\mathrm{N} \mathrm{V}$ and O VI absorption features in Mas-Ribas (2019).

The existence of $\mathrm{O}$ VI, N V, and other line-locking signatures would be best assessed using absorption troughs of these species that are directly identified in the individual quasar spectra instead of using those of C IV. The search for N V and $\mathrm{O}$ VI troughs is difficult, however, because they are in regions of the quasar spectrum that are affected by the Ly $\alpha$ emission line and forest, respectively. Searching for Si IV features may be easier because a fraction of them will likely reside in the line-free region of the rest-frame quasar spectrum. However, the strength of this doublet is significantly weaker than those of O VI, N V, and C IV (Figure 1), and so its line-locking feature could also be weak and difficult to detect. A catalog of Si IV absorption troughs recently built by Guo \& Martini (2019) will enable such a calculation. Alternatively, an autocorrelation analysis in the real BOSS spectra may reveal the characteristic distances at which absorption troughs are located, although this requires obtaining the continuum for each individual quasar spectrum with precision, including the region within the Ly $\alpha$ forest, where the continuum is difficult to obtain (FaucherGiguère et al. 2008a). We defer these calculations to future work.

\subsection{Comparison to Previous Work}

A number of works have previously performed stacks of quasar spectra containing outflows (e.g., Baskin et al. 2013; Hamann et al. 2019). These works have mostly focused on the analysis of the composite absorption troughs, while our work is concerned with the detection of the line-locking signatures. To fit the absorption lines, we have concentrated our attention on the regions where the lines are expected; more specifically, we have disregarded broad absorption wings that can appear on the blue side of some of the strongest absorption lines, and for stacks considering the broadest troughs (Section 3.3.1). Therefore, our stacks can in some cases present shallower and narrower absorption lines than those that were accounted for in previous works.

In addition, our stacking methodology enables us to identify the different components of the atomic doublets in most composites, while these appear less well resolved in other works. The reason behind this effect may arise from the fact that we align the spectra for the stacks considering the position of the minimum flux within the troughs directly, without smoothing the flux to account for the impact of noise. If this is true, this might also be the reason why line-locking has not been detected in most previous works. Only Bowler et al. (2014) have found the signature of line-locking in stacked 
spectra. These authors stacked narrow absorbers $\left(<200 \mathrm{~km} \mathrm{~s}^{-1}\right.$ C IV troughs) in SDSS quasars that contain BALs. Although this implies that the quasars have outflows, they do not address the case of broad absorbers because the line and line-locking features could not be resolved. Thus, our work is the first to demonstrate the presence of line-locking in quasar outflows, from narrow to broad absorbers and for different trough and quasar properties. It is important to mention, however, that Baskin et al. (2013) reported tentative evidence for radiative acceleration in their Appendix B, resulting from the relation between the depth of the absorption profiles and the luminosity of the sources.

\section{Conclusion}

We have built 36 outflow composite spectra by stacking broad $\left(>450 \mathrm{~km} \mathrm{~s}^{-1}\right)$ absorption line systems in the spectra of SDSS-III/BOSS DR12 quasars. We have computed the composites considering bins in outflow velocity, width of the troughs, degree of detachment between the troughs and the quasars, and quasar redshift and brightness. For every spectrum, we modeled the absorption profiles considering the line and line-locking components for a large number of atomic transitions. This atomic data set and the composite outflow spectra are publicly available at https:// github.com/lluism/BALs. Our results can be summarized as follows:

1. An absorption feature is visible on the blue side of all the strong absorption lines and in all our 36 composite spectra. This feature is well fit assuming that it is the C IV line-locking component of the respective absorption lines at a distance of $497 \mathrm{~km} \mathrm{~s}^{-1}$ from them, corresponding to the C IV doublet separation.

2. The detectability of the C IV line-locking feature compared to that of the line does not seem to depend on the $\mathrm{S} / \mathrm{N}$ of the spectra or the depth of the troughs, suggesting that the locking feature is real.

3. We investigate the presence of line-locking features from the doublets of O VI, Si IV, and N V, but these seem to not be present.

4. Our composite spectra resolve the two lines of the C IV and other doublets, although the mean width value of the troughs in the stacks is $\approx 2000 \mathrm{~km} \mathrm{~s}^{-1}$. This implies that (1) the position of the minimum flux in the absorption troughs, which we use for centering our stacks, has a physical meaning (likely representing the blue line of the C IV doublet), and (2) the broad absorption troughs may consist of the superposition of narrow absorbers.

Our results indicate that radiative acceleration is a common mechanism intervening in quasar outflows, and that its presence depends weakly on the characteristics of the winds. Models and simulations assessing the connection between the outflows and AGN feedback should therefore incorporate radiative transfer processes that account for the coupling between radiation and the gas, especially the resonant scattering of photons. The omnipresence of line-locking in different wind conditions also suggests that radiative acceleration might be a dominant effect in other environments. These could be the outflows from gamma-ray bursts (Castro-Tirado et al. 2010), quasar jets (Gravity Collaboration et al. 2017), or Seyfert galaxies, where line-locking has been suggested and tentative detections have been reported. Finally, the fact that line-locking is observed for the C IV doublet but not for other species may be connected to the physical properties of a characteristic multiphase structure in the outflow, where different species trace different regions. We present and test this hypothesis in more detail in a companion paper, MasRibas (2019).

The initial inspiration for this work grew out of a stimulating discussion with Paul Martini during a visit supported by the Visitor Program at the Ohio State Center for Cosmology and Astroparticle Physics. We are grateful to him for valuable ideas and comments on our paper, and to the CCAP for their kind hospitality. We thank the anonymous referee for a detailed revision and a constructive report that helped improve our work. We thank Stan Owocki for sharing with us his thoughts and inspiring notes on the dynamics of CIV line-locking. We also thank Ainar Drews, Mattia Mina, Robert Wissing, and Håvard T. Ihle for discussions on line-locking and statistical aspects, and Tzu-Ching Chang, Olivier Doré Phil Berger, Sterl Phinney, Lee Armus, Ski Antonucci, Brice Ménard, Jordi Miralda Escudé Sijing Shen, Johan Fynbo, Joop Schaye, Jason X. Prochaska, Bill Forman, and Christine Jones for enriching conversations and suggestions. We are grateful to Signe Riemer-Sørensen for many useful discussions on quasar continua. L.M.R. is grateful to the UCSB/MPIA ENIGMA group for their kind hospitality, and together with other colleagues at JPL and Caltech, for many inspiring discussions during this work. This research was partially carried out at the Jet Propulsion Laboratory, California Institute of Technology, under a contract with the National Aeronautics and Space Administration.

Funding for SDSS-III has been provided by the Alfred P. Sloan Foundation, the Participating Institutions, the National Science Foundation, and the U.S. Department of Energy Office of Science. The SDSS-III website is http://www.sdss3.org/. SDSS-III is managed by the Astrophysical Research Consortium for the Participating Institutions of the SDSS-III Collaboration including the University of Arizona, the Brazilian Participation Group, Brookhaven National Laboratory, Carnegie Mellon University, University of Florida, the French Participation Group, the German Participation Group, Harvard University, the Instituto de Astrofisica de Canarias, the Michigan State/Notre Dame/JINA Participation Group, Johns Hopkins University, Lawrence Berkeley National Laboratory, Max Planck Institute for Astrophysics, Max Planck Institute for Extraterrestrial Physics, New Mexico State University, New York University, Ohio State University, Pennsylvania State University, University of Portsmouth, Princeton University, the Spanish Participation Group, University of Tokyo, University of Utah, Vanderbilt University, University of Virginia, University of Washington, and Yale University.

\section{Appendix}

Figure 10 illustrates the fits to the four strongest absorption features in all our composite spectra. Cases for which a good fit cannot be obtained are left as blank. The color code is the same as used in Figure 6. 


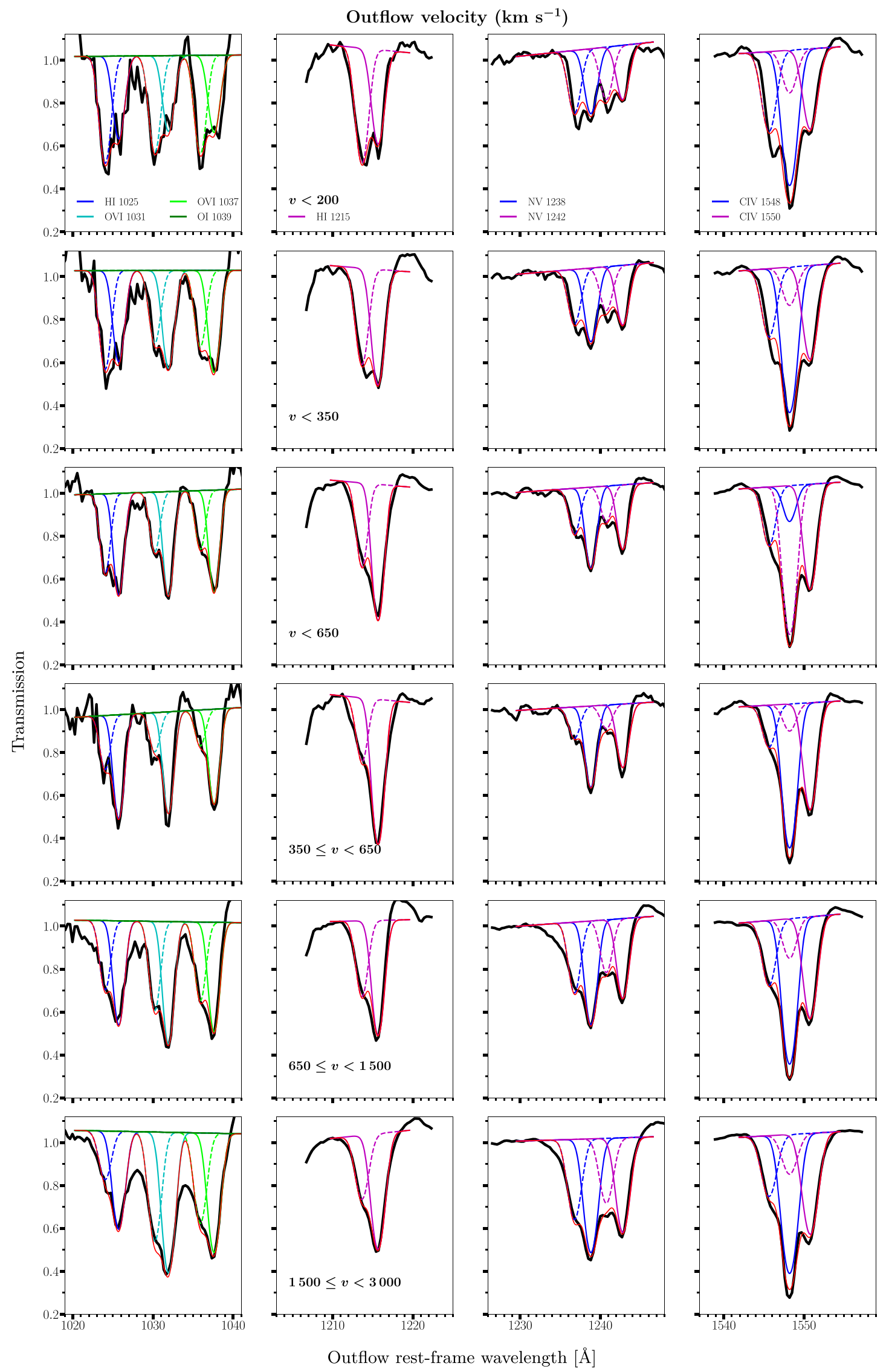

Figure 10. Fits to the strongest features for the composite outflow spectra in Table 2, as in Figure 6. 

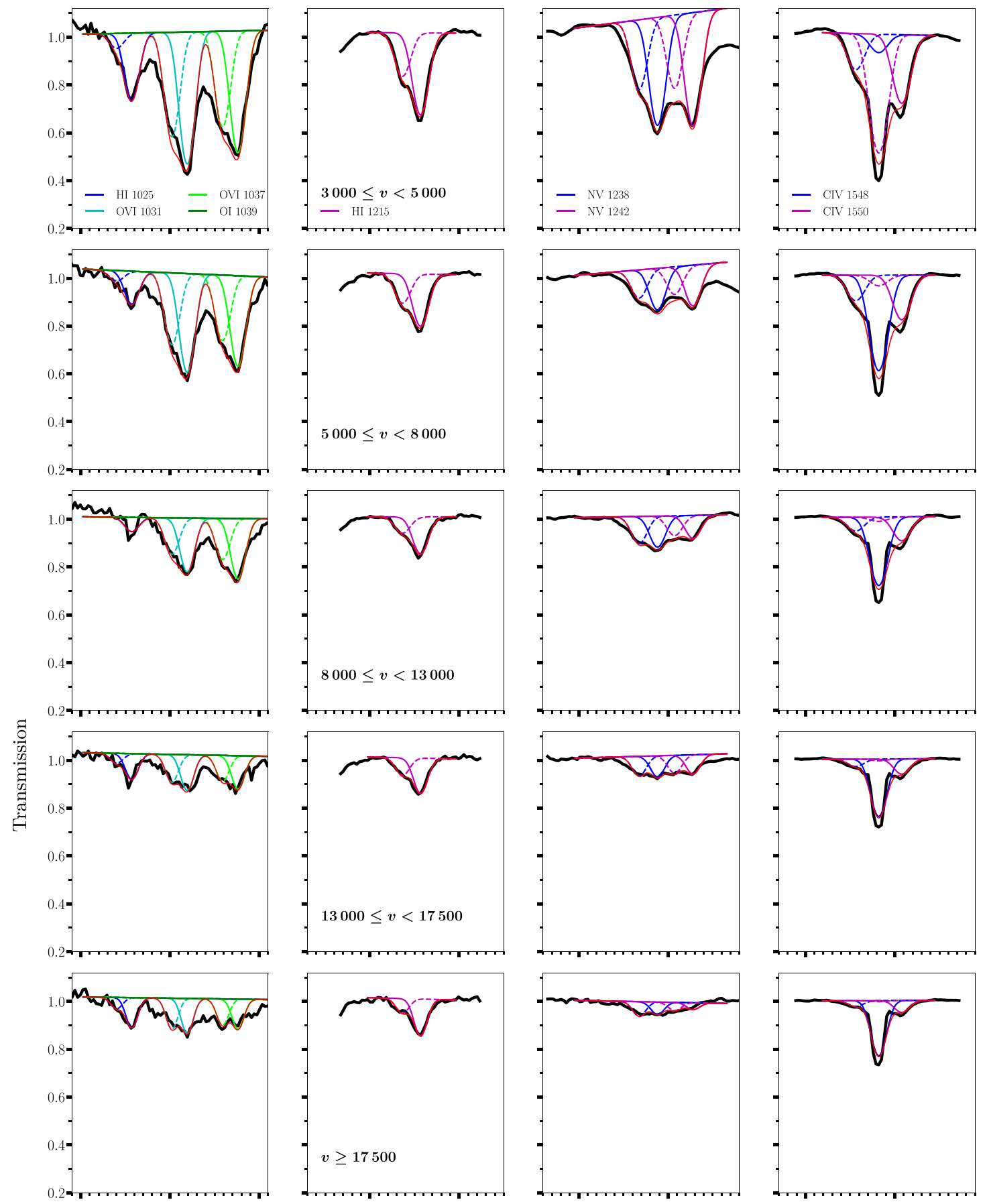

Trough width $\left(\mathrm{km} \mathrm{s}^{-1}\right)$
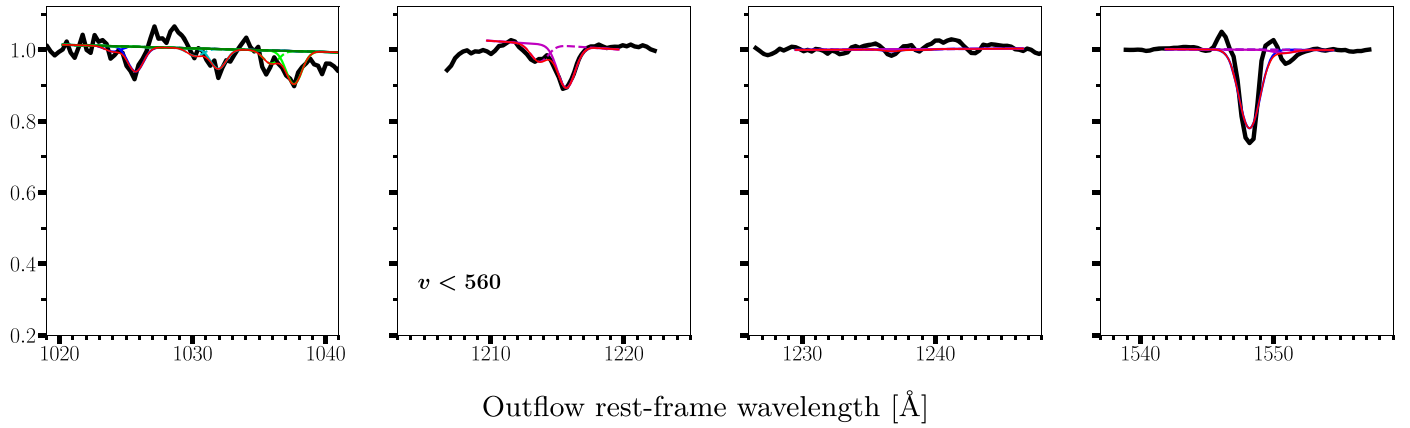

Figure 10. (Continued.) 

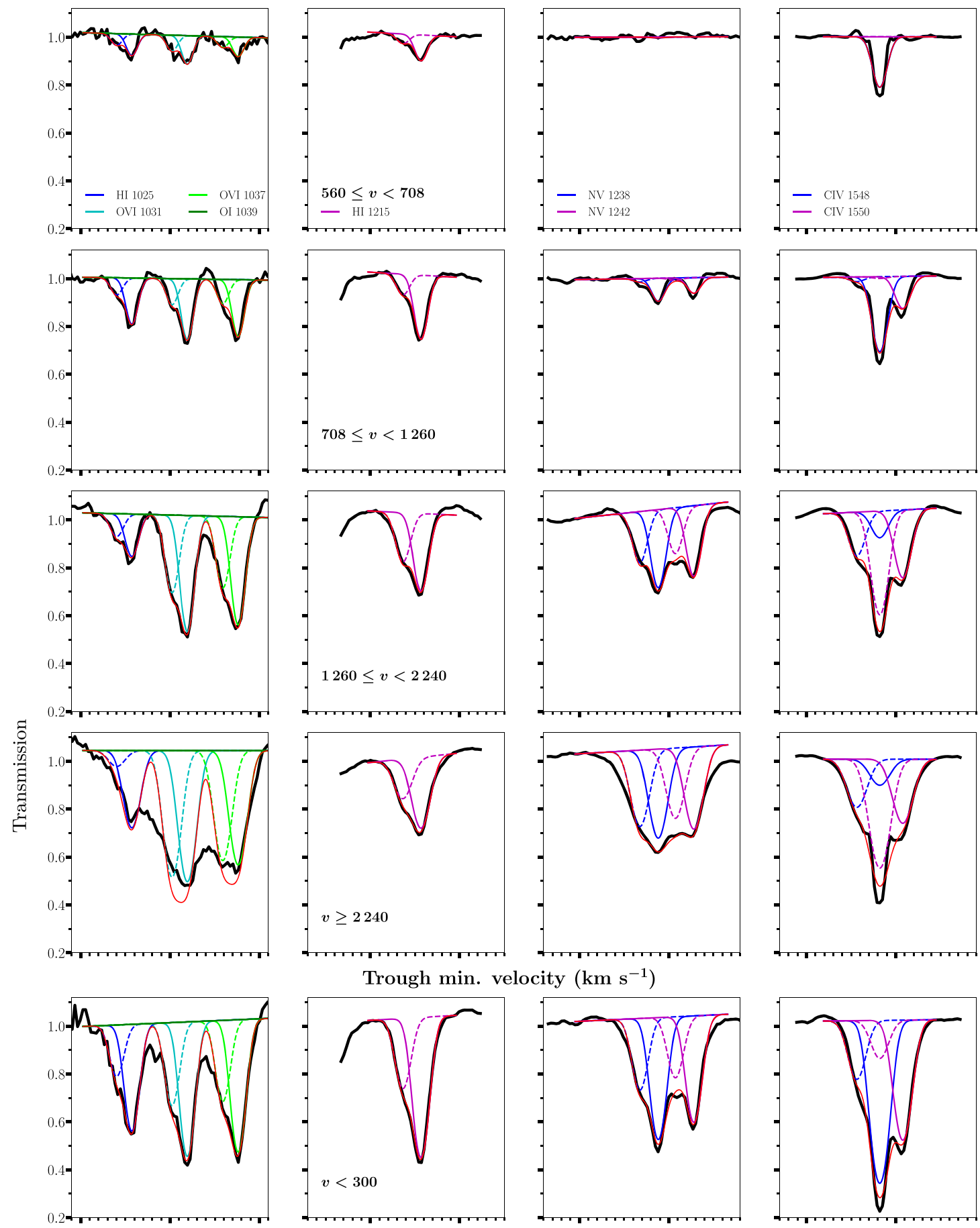

Trough min. velocity $\left(\mathrm{km} \mathrm{s}^{-1}\right)$
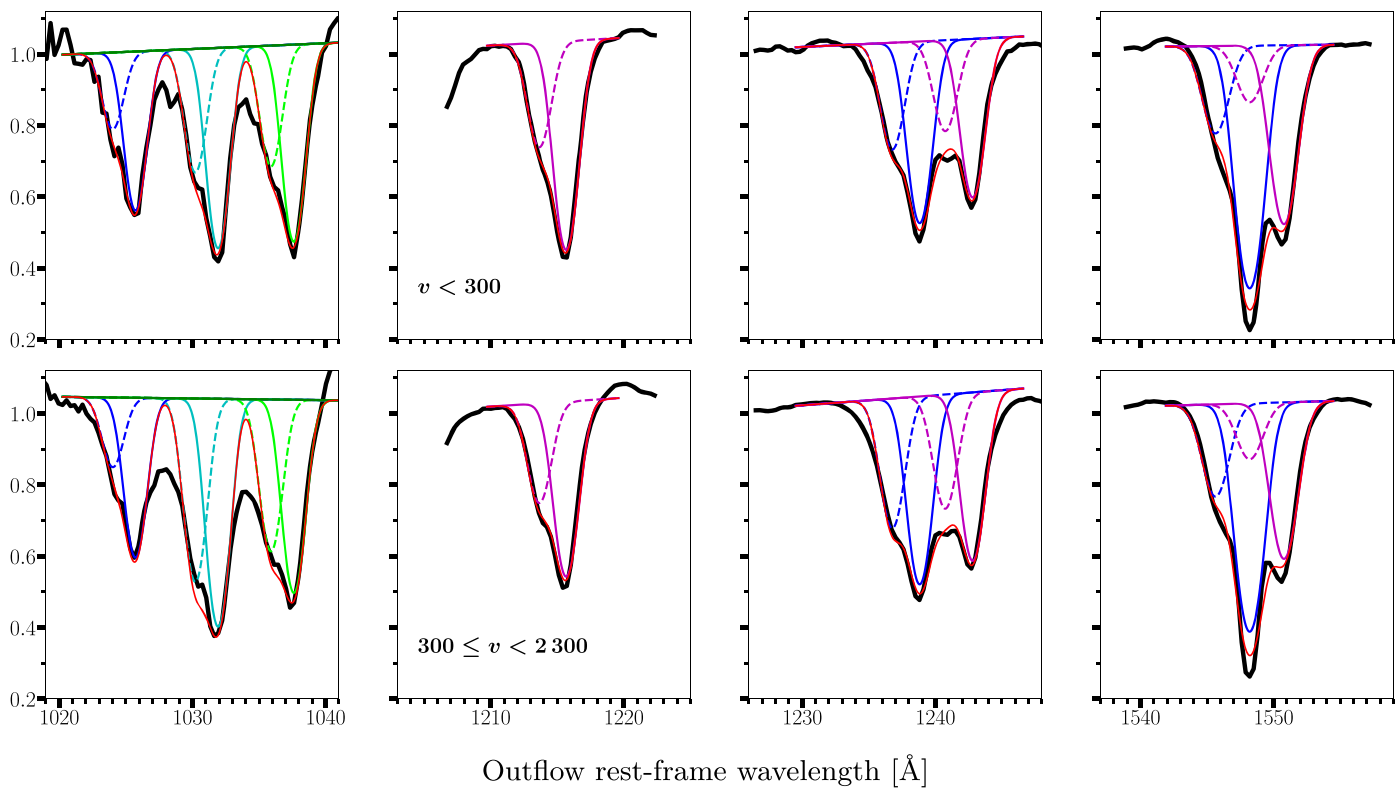

Figure 10. (Continued.) 

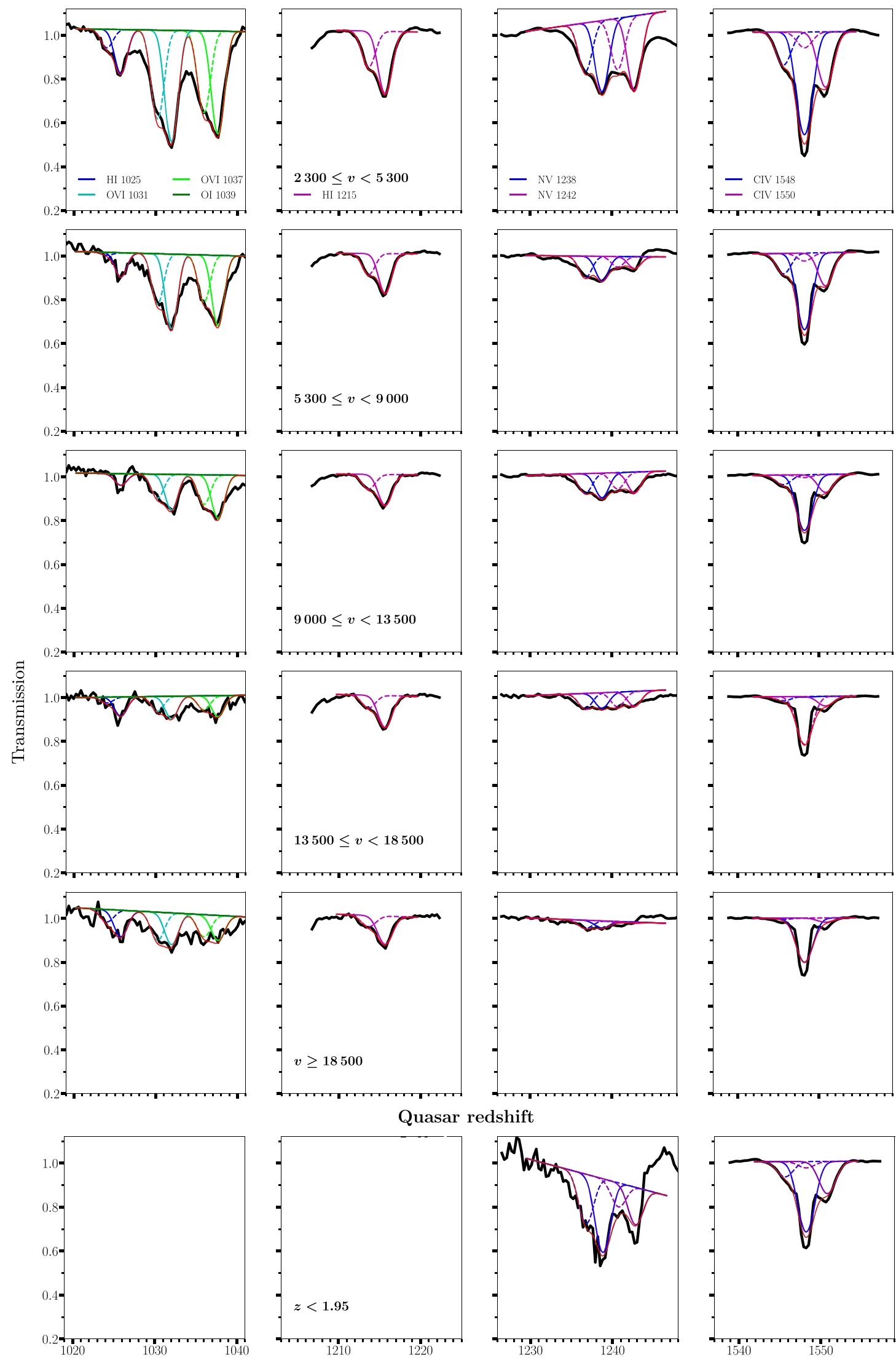

Quasar redshift
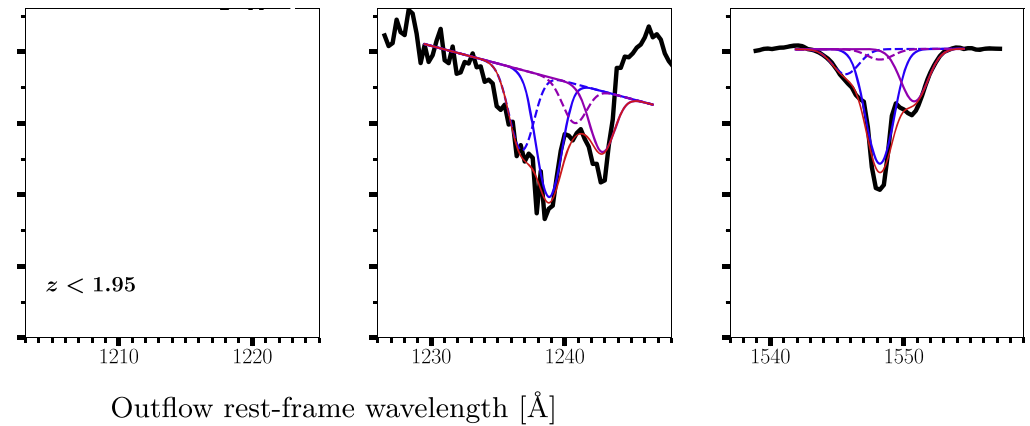

Outflow rest-frame wavelength $[\AA]$

Figure 10. (Continued.) 

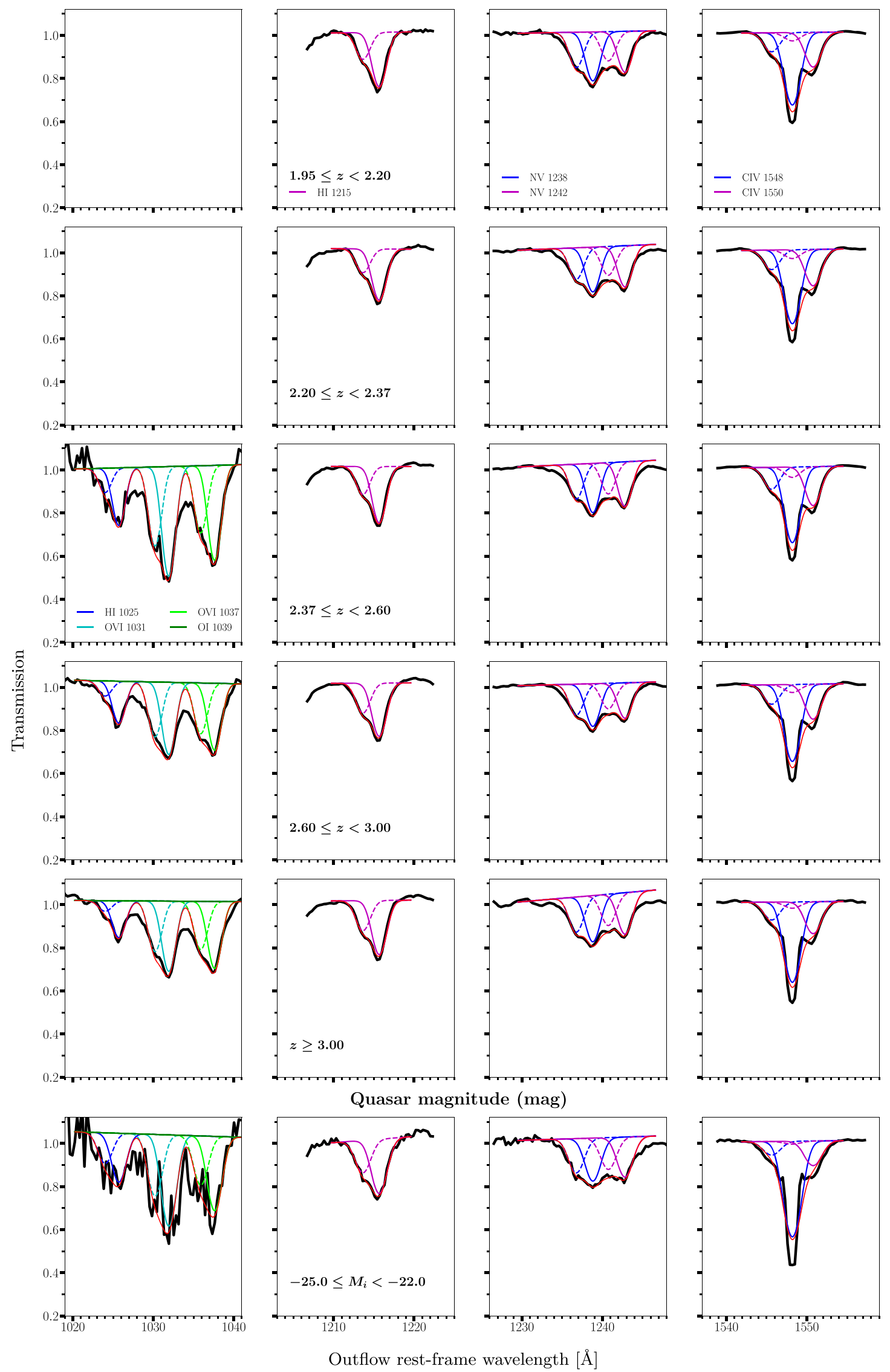

Figure 10. (Continued.) 

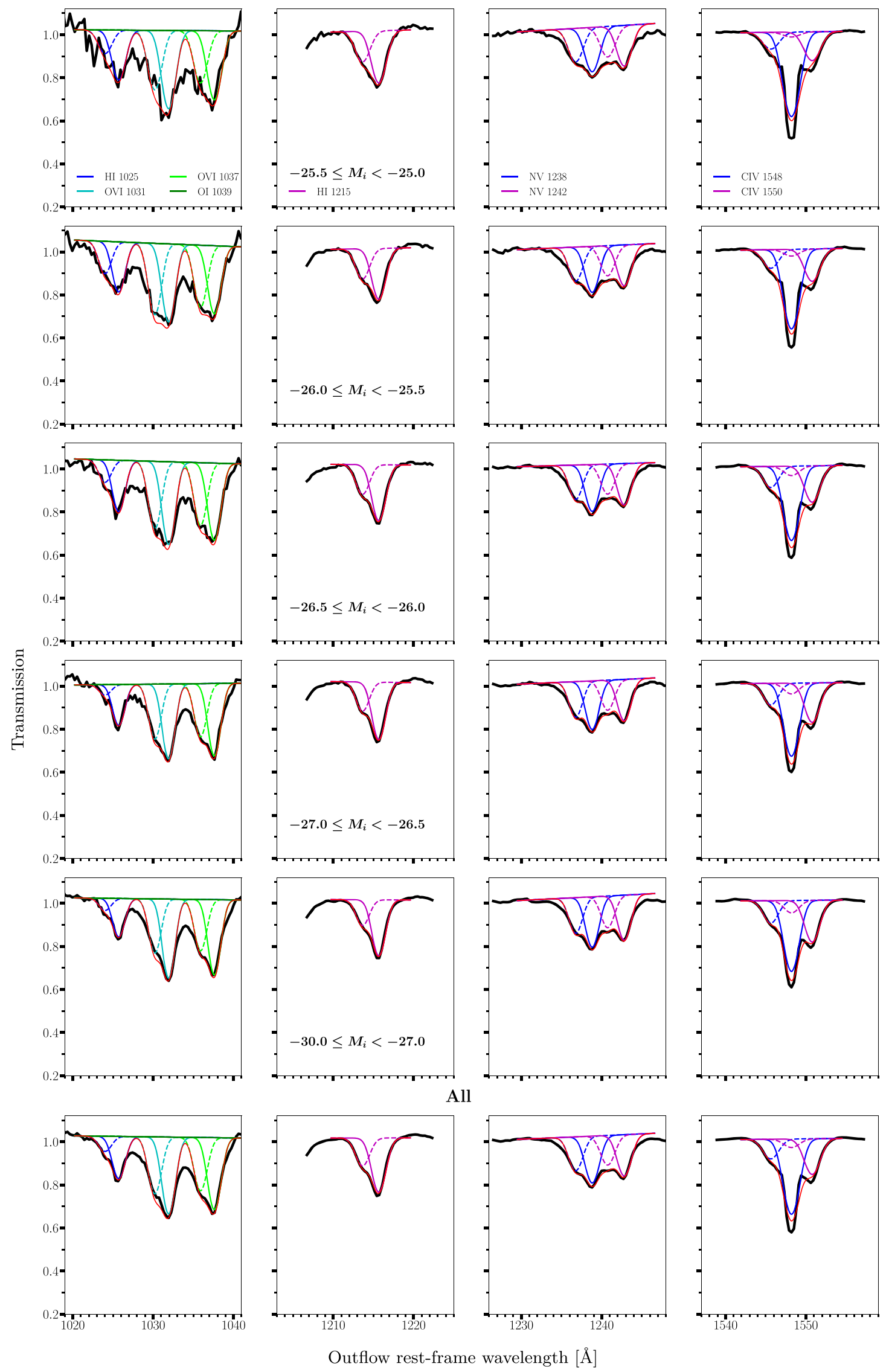

Figure 10. (Continued.) 


\section{ORCID iDs}

Lluís Mas-Ribas (1) https://orcid.org/0000-0003-4584-8841

Renate Mauland (i) https://orcid.org/0000-0003-3385-9447

\section{References}

Arav, N., \& Begelman, M. C. 1994, ApJ, 434, 479

Arav, N., \& Li, Z.-Y. 1994, ApJ, 427, 700

Baldwin, J. A. 1977, ApJ, 214, 679

Barnes, D. J., Kannan, R., Vogelsberger, M., \& Marinacci, F. 2018, arXiv: 1812.01611

Baskin, A., Laor, A., \& Hamann, F. 2013, MNRAS, 432, 1525

Baskin, A., Laor, A., \& Stern, J. 2014, MNRAS, 445, 3025

Bianchi, S., Guainazzi, M., Laor, A., Stern, J., \& Behar, E. 2019, MNRAS, 485, 416

Bowler, R. A. A., Hewett, P. C., Allen, J. T., \& Ferland, G. J. 2014, MNRAS, 445, 359

Braun, E., \& Milgrom, M. 1989, ApJ, 342, 100

Burbidge, E. M., \& Burbidge, G. R. 1975, ApJ, 202, 287

Castro-Tirado, A. J., Møller, P., García-Segura, G., et al. 2010, A\&A, 517, A61

Cavaliere, A., Lapi, A., \& Menci, N. 2002, ApJL, 581, L1

Chelouche, D., \& Netzer, H. 2003, MNRAS, 344, 233

Ciotti, L., Ostriker, J. P., \& Proga, D. 2010, ApJ, 717, 708

Davies, F. B., Hennawi, J. F., Bañados, E., et al. 2018, ApJ, 864, 143

Dawson, K. S., Schlegel, D. J., Ahn, C. P., et al. 2013, AJ, 145, 10

de Kool, M., \& Begelman, M. C. 1995, ApJ, 455, 448

Eisenstein, D. J., Weinberg, D. H., Agol, E., et al. 2011, AJ, 142, 72

Faucher-Giguère, C.-A., Lidz, A., Hernquist, L., \& Zaldarriaga, M. 2008a, ApJ, 688, 85

Faucher-Giguère, C.-A., Prochaska, J. X., Lidz, A., Hernquist, L., \& Zaldarriaga, M. 2008b, ApJ, 681, 831

Foltz, C. B., Weymann, R. J., Morris, S. L., \& Turnshek, D. A. 1987, ApJ, 317,450

Furlanetto, S. R., \& Loeb, A. 2001, ApJ, 556, 619

Gabel, J. R., Arav, N., \& Kim, T.-S. 2006, ApJ, 646, 742

Ganguly, R., Masiero, J., Charlton, J. C., \& Sembach, K. R. 2003, ApJ, 598, 922

Gravity Collaboration, Petrucci, P.-O., Waisberg, I., et al. 2017, A\&A, 602, L11

Gunn, J. E., Carr, M., Rockosi, C., et al. 1998, AJ, 116, 3040

Gunn, J. E., Siegmund, W. A., Mannery, E. J., et al. 2006, AJ, 131, 2332

Guo, Z., \& Martini, P. 2019, ApJ, 879, 72

Gupta, N., Srianand, R., Petitjean, P., \& Ledoux, C. 2003, A\&A, 406, 65

Haiman, Z., \& Bryan, G. L. 2006, ApJ, 650, 7

Hamann, F., Herbst, H., Paris, I., \& Capellupo, D. 2019, MNRAS, 483, 1808

Harrison, C. M. 2017, NatAs, 1, 0165

Harrison, C. M., Costa, T., Tadhunter, C. N., et al. 2018, NatAs, 2, 198
Hopkins, P. F., \& Elvis, M. 2010, MNRAS, 401, 7

King, A., \& Pounds, K. 2015, ARA\&A, 53, 115

Korista, K. T., Voit, G. M., Morris, S. L., \& Weymann, R. J. 1993, ApJS, 88,357

Kramida, A., Ralchenko, Y., Reader, J. \& NIST ASD Team 2018, NIST Atomic Spectra Database (ver. 5.5.6) (Gaithersburg, MD: National Institute of Standards and Technology), https://physics.nist.gov/asd

Laor, A., \& Davis, S. W. 2014, MNRAS, 438, 3024

Leighly, K. M. 2004, ApJ, 611, 125

Levine, R., \& Gnedin, N. Y. 2005, ApJ, 632, 727

Lu, W.-J., \& Lin, Y.-R. 2018a, MNRAS, 474, 3397

Lu, W.-J., \& Lin, Y.-R. 2018b, ApJ, 863, 186

Lucy, L. B., \& Solomon, P. M. 1970, ApJ, 159, 879

Lynds, C. R. 1967, ApJ, 147, 396

Mas-Ribas, L. 2019, arXiv:1903.08170

Mas-Ribas, L., Miralda-Escudé, J., Pérez-Ràfols, I., et al. 2017, ApJ, 846, 4

Matthews, J. H., Knigge, C., Long, K. S., et al. 2016, MNRAS, 458, 293

Milne, E. A. 1926, MNRAS, 86, 459

Moe, M., Arav, N., Bautista, M. A., \& Korista, K. T. 2009, ApJ, 706, 525

Murray, N., Chiang, J., Grossman, S. A., \& Voit, G. M. 1995, ApJ, 451, 498

Mushotzky, R. F., Solomon, P. M., \& Strittmatter, P. A. 1972, ApJ, 174, 7

Nestor, D., Hamann, F., \& Rodriguez Hidalgo, P. 2008, MNRAS, 386, 2055

North, M., Knigge, C., \& Goad, M. 2006, MNRAS, 365, 1057

Palanque-Delabrouille, N., Yèche, C., Borde, A., et al. 2013, A\&A, 559, A85

Pâris, I., Petitjean, P., Aubourg, É., et al. 2012, A\&A, 548, A66

Pâris, I., Petitjean, P., Ross, N. P., et al. 2017, A\&A, 597, A79

Perrotta, S., D’Odorico, V., Hamann, F., et al. 2018, MNRAS, 481, 105

Planck Collaboration, Ade, P. A. R., Aghanim, N., et al. 2016, A\&A, 594, A13

Prochaska, J. X., Tejos, N., Crighton, N., et al. 2016, linetools/linetools: Second major release, Zenodo, doi:10.5281/zenodo. 168270

Proga, D. 2007a, ApJ, 661, 693

Proga, D. 2007b, in ASP Conf. Ser. 373, The Central Engine of Active Galactic Nuclei, ed. L. C. Ho \& J.-W. Wang (San Francisco, CA: ASP), 267

Proga, D., \& Kallman, T. R. 2004, ApJ, 616, 688

Proga, D., Stone, J. M., \& Kallman, T. R. 2000, ApJ, 543, 686

Proga, D., \& Waters, T. 2015, ApJ, 804, 137

Ross, N. P., Myers, A. D., Sheldon, E. S., et al. 2012, ApJS, 199, 3

Scannapieco, E., \& Oh, S. P. 2004, ApJ, 608, 62

Scargle, J. D. 1973, ApJ, 179, 705

Smee, S. A., Gunn, J. E., Uomoto, A., et al. 2013, AJ, 146, 32

Srianand, R. 2000, ApJ, 528, 617

Srianand, R., Petitjean, P., Ledoux, C., \& Hazard, C. 2002, MNRAS, 336, 753

Wang, T., Yang, C., Wang, H., \& Ferland, G. 2015, ApJ, 814, 150

Waters, T., \& Proga, D. 2016, MNRAS, 460, L79

Weymann, R. J., Morris, S. L., Foltz, C. B., \& Hewett, P. C. 1991, ApJ, 373, 23

Williams, R. E. 1972, ApJ, 178, 105 